\section{Monosaccharides: A ToF-SIMS reference spectra database. II. Positive polarity}

\author{
Laetitia Bernard, Rowena Crockett, and Maciej Kawecki ${ }^{\text {a) }}$ \\ Laboratory of Nanoscale Materials Science, Empa, CH-8600 Dübendorf, Switzerland
}

(Received 20 August 2019; accepted 30 October 2019; published 3 December 2019)

The number of time-of-flight secondary ion mass spectrometry studies on biological tissues and cells has strongly increased since the development of primary ion sources that allow not only elemental but also molecular analysis. Substantial fragmentation during ionic bombardment results in a large number of peaks, rendering data analysis complex. Complete and trustable sets of reference spectra for the main biological building blocks, i.e., amino acids, monosaccharides, fatty acids, and nucleotides, are required. This work aims to provide an accurate and extensive library of reference spectra for monosaccharides, measured with the $\mathrm{Bi}_{3}^{+}$primary ion. Here (Paper II), the positive polarity spectra and lists of associated characteristic fragments are presented. Published by the AVS. https://doi.org/10.1116/1.5125103

Keywords: ToF-SIMS, carbohydrate, sugar, monosaccharide, mass spectrometry, fragmentation

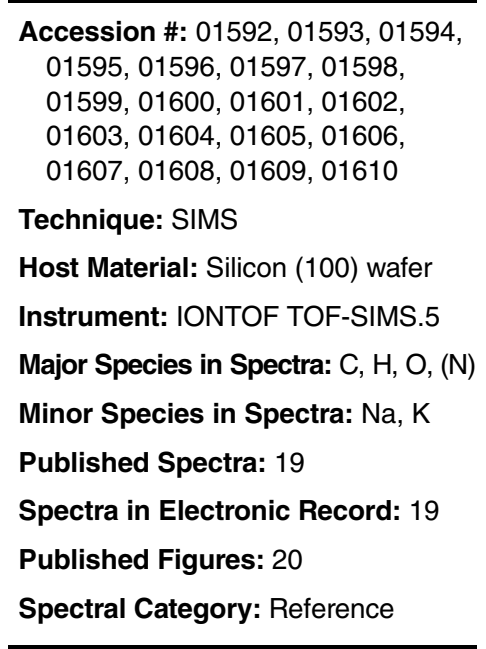

\section{INTRODUCTION}

Monosaccharides are the building blocks of structural polymers of both plant [e.g., cellulose, pectins, and hemicelluloses (Refs. 1 and 2)] and animal origin [e.g., chitin (Refs. 3 and 4)] and are essential components of glycoproteins (Refs. 5-7). Monosaccharides, their derivatives, and polymers are also indispensable in cellular respiration (Ref. 8) and for energy storage and transport in biological organisms (Refs. 9 and 10). Further, specific monosaccharides and monosaccharide derivatives act as secondary messengers [e.g., inositol 1,4,5-triphosphate in $\mathrm{Ca}^{2+}$ release (Ref. 11)], as essential reagents in detoxification [e.g., glucuronic acid in phase II liver metabolism (Ref. 12)], and as markers mediating cell-cell interaction (Ref. 13).

A time-of-flight secondary ion mass spectrometry (ToF-SIMS) study of seven selected hexoses was presented by Berman et al. based on the analysis of positive secondary ions produced by the bombardment with Au+ primary ions (Ref. 14). They could demonstrate the fragmentation pathway of glucose via the loss of successive water molecules. Interestingly, they also found that different isomers undergo different fragmentation patterns, which shows that ToF-SIMS is a powerful tool to obtain an accurate differentiation of monosaccharides. The work presented here provides a library of ToF-SIMS reference spectra for the monosaccharides allose, arabinose, fructose, fucose, galactose, 2-deoxy-galactose, galacturonic acid, glucose, glucuronic acid, mannose, myo-inositol, rhamnose, ribose, 2-deoxy-ribose, xylose, $\mathrm{N}$-acetylgalactosamine, $N$-acetylglucosamine, $\quad N$-acetylmuramic acid, and $\mathrm{N}$-acetylneuraminic acid. Characteristic fragments are listed beside the spectra for each monosaccharide. This part (Paper II) of the monosaccharide database contains the positive polarity spectra. The negative spectra are published in Paper I (Ref. 15). Analog databases for the full set of proteinogenic amino acids + glycine (Refs. 16 and 17) are available. Peak lists (Ref. 18) and reference spectra (Ref. 19) for selected lipids can be found in the literature.

The monosaccharides were purchased in the form of pure powders. D-Galacturonic acid and D-glucuronic acid were from

a)Electronic mail: maciej.kawecki@empa.ch
Fluka and all others from Sigma Aldrich. Each powder was dissolved in freshly de-ionized $\mathrm{H}_{2} \mathrm{O}$ (resistivity $>18.2 \mathrm{M} \Omega \mathrm{cm}$ ) at a concentration of $0.1 \mathrm{M}$. Host silicon wafers of $1 \times 1 \mathrm{~cm}^{2}$ size were first washed by submersion in subsequent ultrasound baths of $2 \times 15 \mathrm{~min}$ in acetone and $1 \times 15 \mathrm{~min}$ in ethanol and then plasma-treated for a duration of $10 \mathrm{~min}$ to render the surface hydrophilic. $50 \mu \mathrm{l}$ of solution was then deposited on the pretreated silicon wafer substrates, and the water was allowed to evaporate in a fume hood at room temperature.

The spectra were acquired on a ToF-SIMS 5 instrument (IONTOF GmbH) with a $25 \mathrm{keV} \mathrm{Bi}_{3}^{+}$primary ion beam operated in high mass resolution spectral mode. The primary ion dose density was kept below the static limit $\left(\leq 10^{12}\right.$ ions $\left./ \mathrm{cm}^{2}\right)$. Four to ten randomly selected areas of $200 \times 200 \mu \mathrm{m}^{2}\left(128 \times 128 \mathrm{pxls}^{2}\right.$, 50 scans) were analyzed for each monosaccharide sample, to insure good reproducibility. No electron flood gun was used. All spectra were normalized to their respective total ions count. The most relevant characteristic fragments are displayed directly on the spectra and reported in the tables. Intact monosaccharide molecules are referred to as " $M$," contaminants as " $c$.," and cationization products as "c.p." Major peaks where a unique attribution was not possible, e.g., due to overlap beyond the mass resolution of two potential fragmentation products, are marked with "n.a." standing for not (uniquely) attributed. The deviation values for all assigned peaks are below $150 \mathrm{ppm}$. The peak assignments to the fragmentation species given in the tables are tentative, and the smaller fragments are nonspecific. The unfragmented molecular ion $[\mathrm{M}+\mathrm{H}]^{+}$displays a low signal-to-noise ratio for all monosaccharides except amino sugars and myo-inositol. All monosaccharides show strong $\left[\mathrm{M}-\mathrm{n}\left(\mathrm{H}_{2} \mathrm{O}\right)+\mathrm{H}\right]^{+}$peaks.

Raw data (ASCII) are included in the supplementary material (Ref. 20).

\section{SPECIMEN DESCRIPTION}

Accession \#: 01592, 01593, 01594, 01595, 01596, 01597, 01598, 01599, 01600, 01601, 01602, 01603, 01604, 01605, 01606, 01607, 01608, 01609, 01610

Host Material: Silicon (100) wafer

CAS Registry \#: D-Allose: 2595-97-3, L-arabinose: 87-72-9, D-fructose: 57-48-7, D-fucose: 3615-37-0, D-galactose: 59-23-4, 
2-deoxy-D-galactose: 1949-89-9, D-galacturonic acid: 91510-52-2, D-glucose: 50-99-7, D-glucoronic acid: 6556-12-3, D-mannose: 3458-28-4, myo-inositol: 87-89-8, L-rhamnose monohydrate: 10030-85-0, D-ribose: 50-69-1, 2-deoxy-D-ribose: 533-67-5, D-xylose: $58-86-6, \quad N$-acetyl-D-galactosamine: 1811-31-0, $\mathrm{N}$-acetyl-D-glucosamine: 7512-17-6, $\mathrm{N}$-acetylmuramic acid: 10597-89-4, $N$-acetylneuraminic acid: $131-48-6$

Host Material Characteristics: Homogeneous; solid; single crystal; semiconductor

Chemical Name: D-Allose (01592), L-arabinose (01593), D-fructose (01594), D-fucose (01595), D-galactose (01596), 2-deoxy-D-galactose (01597), D-galacturonic acid (01598), D-glucose (01599), D-glucuronic acid (01600), D-mannose (01601), myo-inositol (01602), L-rhamnose (01603), D-ribose (01604), 2-deoxy-D-ribose (01605), D-xylose (01606), $\mathrm{N}$-acetyl-D-galactosamine (01607), $\mathrm{N}$-acetyl-D-glucosamine (01608), $\mathrm{N}$-acetylmuramic acid (01609), $\mathrm{N}$-acetylneuraminic acid (01610)

Source: Sigma Aldrich and Fluka

Host Composition: ${ }_{\text {nox }} \mathrm{SiO}_{2} / \mathrm{SiO}_{2}$

Form: Pure powder dissolved in freshly de-ionized $\mathrm{H}_{2} \mathrm{O}$, sonicated and subsequently drop-deposited on the host wafer

Lot No.: Not specified

Structure: Allose: $\mathrm{C}_{6} \mathrm{H}_{12} \mathrm{O}_{6}$, arabinose: $\mathrm{C}_{5} \mathrm{H}_{10} \mathrm{O}_{5}$, fructose: $\mathrm{C}_{6} \mathrm{H}_{12} \mathrm{O}_{6}$, fucose: $\mathrm{C}_{6} \mathrm{H}_{12} \mathrm{O}_{5}$, galactose: $\mathrm{C}_{6} \mathrm{H}_{12} \mathrm{O}_{6}, \quad$ 2-deoxy-galactose: $\mathrm{C}_{6} \mathrm{H}_{12} \mathrm{O}_{5}$, galacturonic acid: $\mathrm{C}_{6} \mathrm{H}_{10} \mathrm{O}_{7}$, glucose: $\mathrm{C}_{6} \mathrm{H}_{12} \mathrm{O}_{6}$, glucoronic acid: $\mathrm{C}_{6} \mathrm{H}_{10} \mathrm{O}_{7}$, mannose: $\mathrm{C}_{6} \mathrm{H}_{12} \mathrm{O}_{6}$, myo-inositol: $\mathrm{C}_{6} \mathrm{H}_{12} \mathrm{O}_{6}$, rhamnose: $\mathrm{C}_{6} \mathrm{H}_{12} \mathrm{O}_{5}$, ribose: $\mathrm{C}_{5} \mathrm{H}_{10} \mathrm{O}_{5}$, 2-deoxy-ribose: $\mathrm{C}_{5} \mathrm{H}_{10} \mathrm{O}_{4}$, xylose: $\mathrm{C}_{5} \mathrm{H}_{10} \mathrm{O}_{5}, \quad N$-acetylgalactosamine: $\mathrm{C}_{8} \mathrm{H}_{15} \mathrm{NO}_{6}$, $N$-acetylglucosamine: $\mathrm{C}_{8} \mathrm{H}_{15} \mathrm{NO}_{6}, \mathrm{~N}$-acetylmuramic acid: $\mathrm{C}_{11} \mathrm{H}_{19} \mathrm{NO}_{8}, \mathrm{~N}$-acetylneuraminic acid: $\mathrm{C}_{11} \mathrm{H}_{19} \mathrm{NO}_{9}$

History and Significance: As such and/or combined to other small molecules, monosaccharides play various essential roles in the structure and metabolic functions of living organisms.

As Received Condition: Pure powders in glass vials

Analyzed Region: 4-10 randomly selected regions of $200 \times 200 \mu \mathrm{m}^{2}$

Ex Situ Preparation/Mounting: $50 \mu \mathrm{l}$ of $0.1 \mathrm{M}$ monosaccharide solutions, prepared in freshly de-ionized $\mathrm{H}_{2} \mathrm{O}$, was dropdeposited on $1 \times 1 \mathrm{~cm}^{2}$ plasma-treated $\mathrm{Si}$ (100) substrates, in a fume hood. Full coverage of the solutions on each wafer surface was assured. The samples were left in the fume hood for drying and then directly inserted in the UHV chamber and analyzed.

In Situ Preparation: None

Charge Control: None

Temp. During Analysis: $300 \mathrm{~K}$

Pressure During Analysis: $5.6 \times 10^{-8} \mathrm{~Pa}$

Preanalysis Beam Exposure: None

\section{INSTRUMENT CONFIGURATION}

Manufacturer and Model: IONTOF TOF-SIMS.5

Analyzer Type: Time-of-flight

Detector Type: Microchannel plate

Experiment Type: Mass spectra
Sample Rotation: None

Oxygen Flood Source: None

Other Flood Source: None

Unique Instrument Features Used: None

Energy Acceptance Window: $20 \mathrm{eV}$

Postacceleration Voltage: $10000 \mathrm{eV}$

Sample Bias: $0 \mathrm{eV}$

Specimen Normal-to-analyzer $(\Theta e): 0^{\circ}$

\section{Ion Sources}

Ion Source 1 of 1

Purpose of this Ion Source: Analysis beam

Ion Source Manufacturer: IONTOF (Münster, Germany)

Ion Source Model: $25 \mathrm{keV} \mathrm{Bi/Mn} \mathrm{emitter}$

Beam Mass Filter: Electrodynamic mass filter

Beam Species and Charge State: $\mathrm{Bi}_{3}^{+}$

Beam Gating Used: None

Additional Beam Comments: None

Beam Voltage: $25000 \mathrm{eV}$

Net Beam Voltage (impact voltage): $25000 \mathrm{eV}$

Ion Pulse Width: $17.2 \mathrm{~ns}$

Ion Pulse Rate: $10 \mathrm{kHz}$

Pulsed Beam Current: $0.00074-0.00079$ nA

Current Measurement Method: Faraday cup

Beam Diameter: N/A $(\mu \mathrm{m})$

Beam Raster Size: $200 \times 200 \mu \mathrm{m}^{2}$

Beam Incident Angle: $45^{\circ}$

Source-to-Analyzer Angle: $45^{\circ}$

\section{ACKNOWLEDGMENT}

The authors are thankful for the funding received from the Swiss National Science Foundation through Grant No. CR23I2-162828.

\section{REFERENCES}

1. H. Höfte and A. Voxeur, Curr. Biol. 27, 853 (2017).

2. H. Zhu, W. Luo, P. N. Ciesielski, Z. Fang, J. Y. Zhu, G. Henriksson, M. E. Himmel, and L. Hu, Chem. Rev. 116, 9305 (2016).

3. H.-O. Fabritius, C. Sachs, P. R. Triguero, and D. Raabe, Adv. Mater. 21, 391 (2009).

4. N. V. Majeti and R. Kumar, React. Funct. Polym. 46, 1 (2000).

5. C. G. Gahmberg and M. Tolvanen, Trends Bichem. Sci. 21, 308 (1996).

6. G. J. Strous and J. Dekker, Crit. Rev. Biochem. Mol. Biol. 27, 57 (1992).

7. E. Maverakis, K. Kim, M. Shimoda, M. E. Gershwin, F. Patel, R. Wilken, S. Raychaudhuri, L. R. Ruhaak, and C. B. Lebrilla, J. Autoimmun. 57, 1 (2015). 
8. B. Alberts, A. Johnson, J. Lewis, D. Morgan, M. Raff, K. Roberts, and P. Walter, Molecular Biology of the Cell, 6th ed. (Garland Science, New York, 2008), Chap. 2.

9. S. C. Zeeman, J. Kossmann, and A. M. Smith, Annu. Rev. Plant. Biol. 61, 209 (2010).

10. D. H. Wasserman, Am. J. Physiol. Endocrinol. Metab. 296, E11 (2009).

11. H. Streb, R. F. Irvine, M. J. Berridge, and I. Schulz, Nature 306, 67 (1983).

12. G. J. F. Dutton, Glucuronidation of Drugs and Other Compounds (CRC, Boca Raton, FL, 1980).

13. R. L. Schnaar, Arch. Biochem. Biophys. 426, 163 (2004).
14. E. S. F. Berman, K. S. Kulp, M. G. Knize, L. Wu, E. J. Nelson, D. O. Nelson, and K. J. Wu, Anal. Chem. 78, 6497 (2006).

15. L. Bernard, R. Crockett, and M. Kawecki, Surf. Sci. Spectra 26, 025001 (2019).

16. M. Kawecki and L. Bernard, Surf. Sci. Spectra 25, 015001 (2018).

17. M. Kawecki and L. Bernard, Surf. Sci. Spectra 25, 015002 (2018).

18. M. K. Passarelli and N. Winograd, Biochim. Biophys. Acta 1811, 976 (2011).

19. M. J. Taylor, K. Y. Zhang, D. J. Graham, and L. J. Gamble, Surf. Sci. Spectra 25, 025001 (2019).

20. See supplementary material at https://doi.org/10.1116/ 1.5125103 for the raw data exported in ASCII. 


\begin{tabular}{lccc}
\hline & & D-ALLOSE & \\
\hline Spectrum ID \# & Mass (Da) & Species & Peak Assignment \\
\hline 01592 & 73.028 & $\mathrm{C}_{3} \mathrm{H}_{5} \mathrm{O}_{2}{ }^{+}$ & $\mathrm{C} 4$ to $\mathrm{C} 6$ fragment of $\mathrm{M}-\left(\mathrm{H}_{2} \mathrm{O}\right)$ \\
$\ldots$ & 85.028 & $\mathrm{C}_{4} \mathrm{H}_{5} \mathrm{O}_{2}{ }^{+}$ & $\mathrm{C} 3$ to $\mathrm{C} 6$ fragment of $\mathrm{M}-2\left(\mathrm{H}_{2} \mathrm{O}\right)$ \\
$\ldots$ & 97.028 & $\mathrm{C}_{5} \mathrm{H}_{5} \mathrm{O}_{2}{ }^{+}$ & $\mathrm{C} 2$ to $\mathrm{C}$ fragment of $\mathrm{M}-3\left(\mathrm{H}_{2} \mathrm{O}\right)$ \\
$\ldots$ & 103.039 & $\mathrm{C}_{4} \mathrm{H}_{7} \mathrm{O}_{3}{ }^{+}$ & $\mathrm{C}$ to C6 fragment of $\mathrm{M}-\left(\mathrm{H}_{2} \mathrm{O}\right)$ \\
$\ldots$ & 115.039 & $\mathrm{C}_{5} \mathrm{H}_{7} \mathrm{O}_{3}{ }^{+}$ & $\mathrm{C} 2$ to $\mathrm{C} 6$ fragment of $\mathrm{M}-2\left(\mathrm{H}_{2} \mathrm{O}\right)$ \\
$\ldots$ & 127.039 & $\mathrm{C}_{6} \mathrm{H}_{7} \mathrm{O}_{3}{ }^{+}$ & $\mathrm{M}-3\left(\mathrm{H}_{2} \mathrm{O}\right)+\mathrm{H}$ \\
$\ldots$ & 145.050 & $\mathrm{C}_{6} \mathrm{H}_{9} \mathrm{O}_{4}{ }^{+}$ & $\mathrm{M}-2\left(\mathrm{H}_{2} \mathrm{O}\right)+\mathrm{H}$ \\
$\ldots$ & 163.060 & $\mathrm{C}_{6} \mathrm{H}_{11} \mathrm{O}_{5}{ }^{+}$ & $\mathrm{M}-\left(\mathrm{H}_{2} \mathrm{O}\right)+\mathrm{H}$ \\
\hline
\end{tabular}

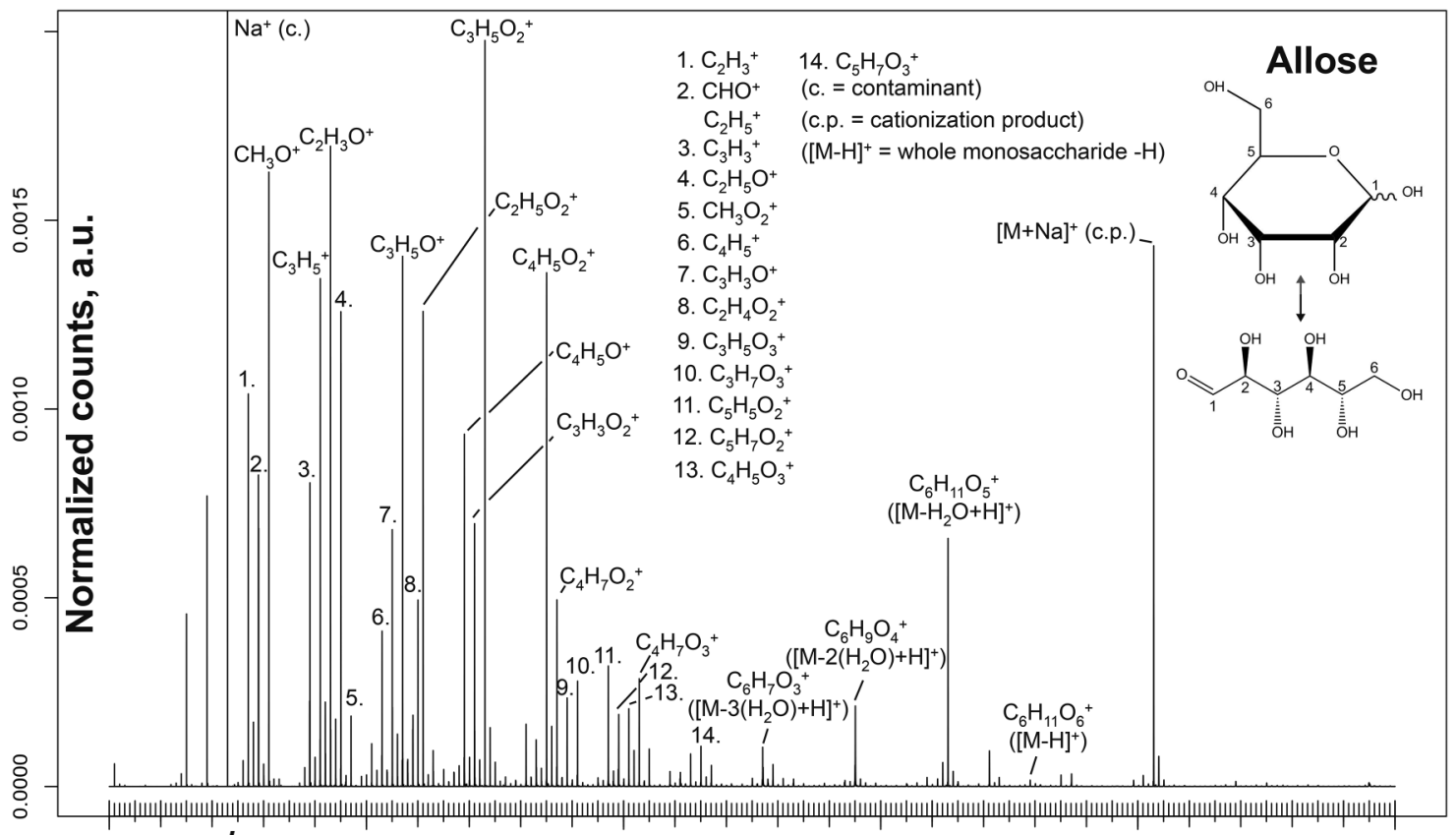

$\begin{array}{llllll}0 & \mathbf{m} / \mathbf{z} & 50 & 100 & 150 & 200\end{array}$

\begin{tabular}{rc}
\hline Accession \#: & $01592-01$ \\
\hline Host Material: & Silicon wafer \\
$\mathbf{\square}$ Technique: & SIMS \\
Secondary Source Polarity: & Positive \\
Mass Range: & $0-250 \mathrm{Da}$ \\
Species Used for Mass Calibration: & $\mathrm{CH}_{2}^{+}, \mathrm{CH}_{3}^{+}, \mathrm{C}_{2} \mathrm{H}_{4}^{+}, \mathrm{C}_{3} \mathrm{H}_{6}{ }^{+}, \mathrm{C}_{2} \mathrm{H}_{4} \mathrm{O}^{+}$ \\
Primary lon Dose: & $9.71 \times 10^{11} \mathrm{~cm}^{-2}$ \\
Primary lon Pulse Width: & $17.2 \mathrm{~ns}^{+}$ \\
Pulsed Beam Current: & $0.00076 \mathrm{nA}$ \\
Biological Significance: & D-Allose exists only in small amounts in nature
\end{tabular}




\begin{tabular}{lccc}
\hline & & L-ARABINOSE & \\
\hline Spectrum ID \# & Mass (Da) & Species & Peak Assignment \\
\hline 01593 & 73.028 & $\mathrm{C}_{3} \mathrm{H}_{5} \mathrm{O}_{2}{ }^{+}$ & $\mathrm{C} 4$ to $\mathrm{C} 6$ fragment of $\mathrm{M}-\left(\mathrm{H}_{2} \mathrm{O}\right)$ \\
$\ldots$ & 85.028 & $\mathrm{C}_{4} \mathrm{H}_{5} \mathrm{O}_{2}{ }^{+}$ & $\mathrm{C} 3$ to $\mathrm{C} 6$ fragment of $\mathrm{M}-2\left(\mathrm{H}_{2} \mathrm{O}\right)$ \\
$\ldots$ & 87.044 & $\mathrm{C}_{4} \mathrm{H}_{7} \mathrm{O}_{2}{ }^{+}$ & - \\
$\ldots$ & 89.023 & $\mathrm{C}_{3} \mathrm{H}_{5} \mathrm{O}_{3}{ }^{+}$ & $\mathrm{C} 1$ to $\mathrm{C} 3$ fragment of $\mathrm{M}+\mathrm{H}$ \\
$\ldots$ & 91.039 & $\mathrm{C}_{3} \mathrm{H}_{7} \mathrm{O}_{3}{ }^{+}$ & $\mathrm{C} 3$ to $\mathrm{C} 5$ fragment of $\mathrm{M}$ \\
$\ldots$ & 97.028 & $\mathrm{C}_{5} \mathrm{H}_{5} \mathrm{O}_{2}{ }^{+}$ & $\mathrm{M}-3\left(\mathrm{H}_{2} \mathrm{O}\right)+\mathrm{H}$ \\
$\ldots$ & 99.044 & $\mathrm{C}_{5} \mathrm{H}_{7} \mathrm{O}_{2}{ }^{+}$ & $\mathrm{M}-3\left(\mathrm{H}_{2} \mathrm{O}\right)+3 \mathrm{H}$ \\
$\ldots$ & 103.039 & $\mathrm{C}_{4} \mathrm{H}_{7} \mathrm{O}_{3}{ }^{+}$ & $\mathrm{C} 1$ to $\mathrm{C} 4$ fragment of M \\
$\ldots$ & 115.039 & $\mathrm{C}_{5} \mathrm{H}_{7} \mathrm{O}_{3}{ }^{+}$ & $\mathrm{M}-2\left(\mathrm{H}_{2} \mathrm{O}\right)+\mathrm{H}$ \\
& 133.050 & $\mathrm{C}_{5} \mathrm{H}_{9} \mathrm{O}_{4}{ }^{+}$ & $\mathrm{M}-\left(\mathrm{H}_{2} \mathrm{O}\right)+\mathrm{H}$ \\
\hline
\end{tabular}

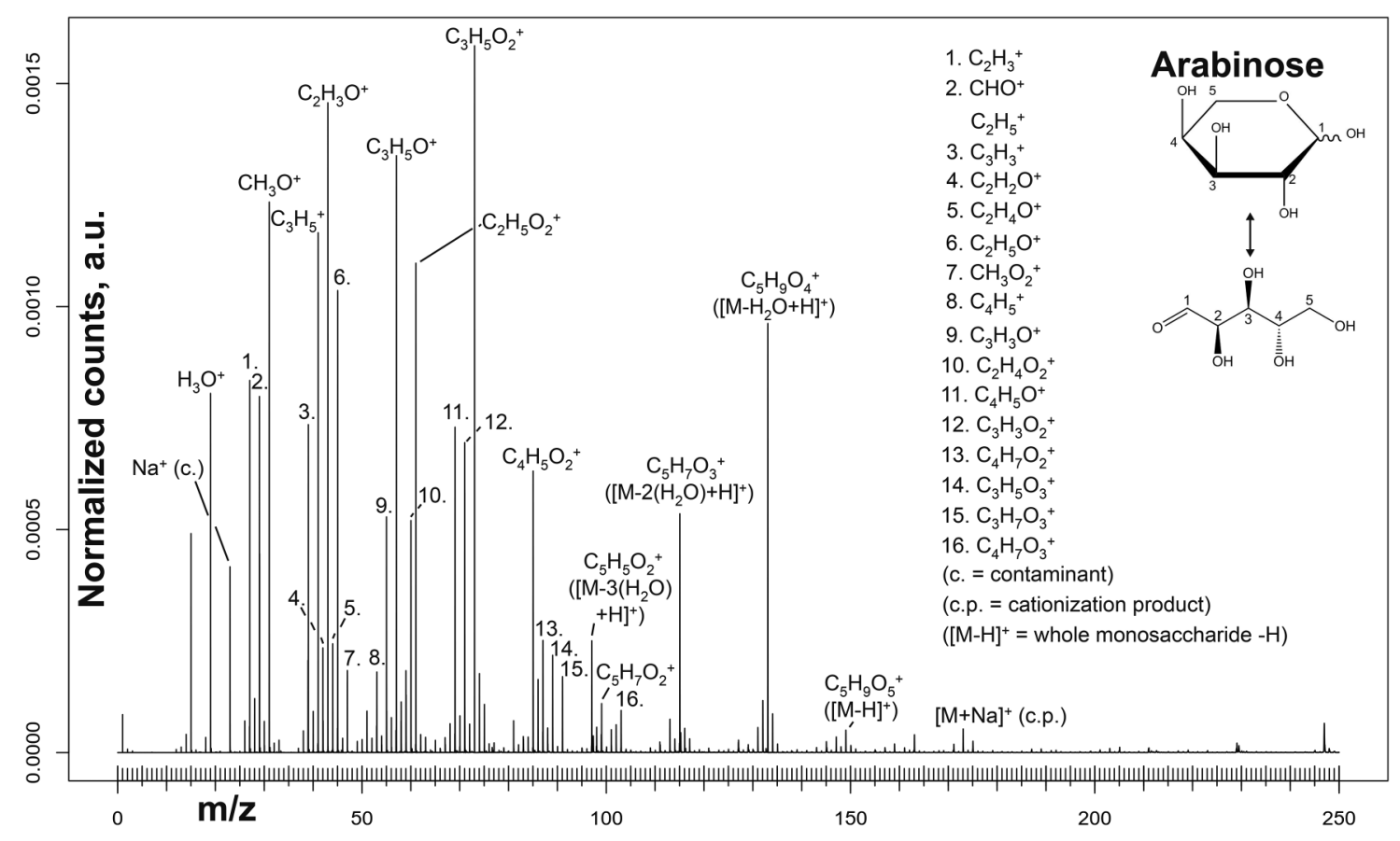

\begin{tabular}{rc}
\hline Accession \#: & $01593-01$ \\
\hline Host Material: & Tilicon wafer \\
Technique: & SIMS \\
Mass Range: & Positive \\
Secondary Source Polarity: & $0-250 \mathrm{Da}$ \\
Species Used for Mass Calibration: & $\mathrm{CH}_{2}^{+}, \mathrm{CH}_{3}^{+}, \mathrm{C}_{2} \mathrm{H}_{4}^{+}, \mathrm{C}_{3} \mathrm{H}_{6}{ }^{+}, \mathrm{C}_{2} \mathrm{H}_{4} \mathrm{O}^{+}$ \\
Primary lon Dose: & $9.71 \times 10^{11} \mathrm{~cm}^{-2}$ \\
Primary lon Pulse Width: & $17.2 \mathrm{~ns}$ \\
Pulsed Beam Current: & $0.00076 \mathrm{nA}$ \\
Biological Significance: & L-Arabinose is found in a range of plant polysaccharides, e.g., the \\
& plant cell wall heteropolysaccharides pectin and hemicellulose \\
\hline
\end{tabular}




\begin{tabular}{|c|c|c|c|}
\hline \multicolumn{4}{|c|}{ D-FRUCTOSE } \\
\hline Spectrum ID \# & Mass (Da) & Species & Peak Assignment \\
\hline 01594 & 73.028 & $\mathrm{C}_{3} \mathrm{H}_{5} \mathrm{O}_{2}^{+}$ & $\mathrm{C} 4$ to $\mathrm{C} 6$ fragment of $\mathrm{M}-\left(\mathrm{H}_{2} \mathrm{O}\right)$ \\
\hline$\ldots$ & 85.028 & $\mathrm{C}_{4} \mathrm{H}_{5} \mathrm{O}_{2}^{+}$ & C3 to $\mathrm{C} 6$ fragment of $\mathrm{M}-2\left(\mathrm{H}_{2} \mathrm{O}\right)$ \\
\hline$\ldots$ & 97.028 & $\mathrm{C}_{5} \mathrm{H}_{5} \mathrm{O}_{2}^{+}$ & $\mathrm{C} 2$ to $\mathrm{C} 6$ fragment of $\mathrm{M}-3\left(\mathrm{H}_{2} \mathrm{O}\right)$ \\
\hline$\ldots$ & 101.023 & $\mathrm{C}_{4} \mathrm{H}_{5} \mathrm{O}_{3}^{+}$ & C3 to $\mathrm{C} 6$ fragment of $\mathrm{M}-\left(\mathrm{H}_{2} \mathrm{O}\right)-2 \mathrm{H}$ \\
\hline$\ldots$ & 103.039 & $\mathrm{C}_{4} \mathrm{H}_{7} \mathrm{O}_{3}^{+}$ & C3 to $\mathrm{C} 6$ fragment of $\mathrm{M}-\left(\mathrm{H}_{2} \mathrm{O}\right)$ \\
\hline$\ldots$ & 115.039 & $\mathrm{C}_{5} \mathrm{H}_{7} \mathrm{O}_{3}^{+}$ & $\mathrm{C} 2$ to $\mathrm{C} 6$ fragment of $\mathrm{M}-2\left(\mathrm{H}_{2} \mathrm{O}\right)$ \\
\hline$\ldots$ & 127.039 & $\mathrm{C}_{6} \mathrm{H}_{7} \mathrm{O}_{3}^{+}$ & $\mathrm{M}-3\left(\mathrm{H}_{2} \mathrm{O}\right)+\mathrm{H}$ \\
\hline$\ldots$ & 145.050 & $\mathrm{C}_{6} \mathrm{H}_{9} \mathrm{O}_{4}^{+}$ & $\mathrm{M}-2\left(\mathrm{H}_{2} \mathrm{O}\right)+\mathrm{H}$ \\
\hline$\ldots$ & 163.060 & $\mathrm{C}_{6} \mathrm{H}_{11} \mathrm{O}_{5}^{+}$ & $\mathrm{M}-\left(\mathrm{H}_{2} \mathrm{O}\right)+\mathrm{H}$ \\
\hline
\end{tabular}

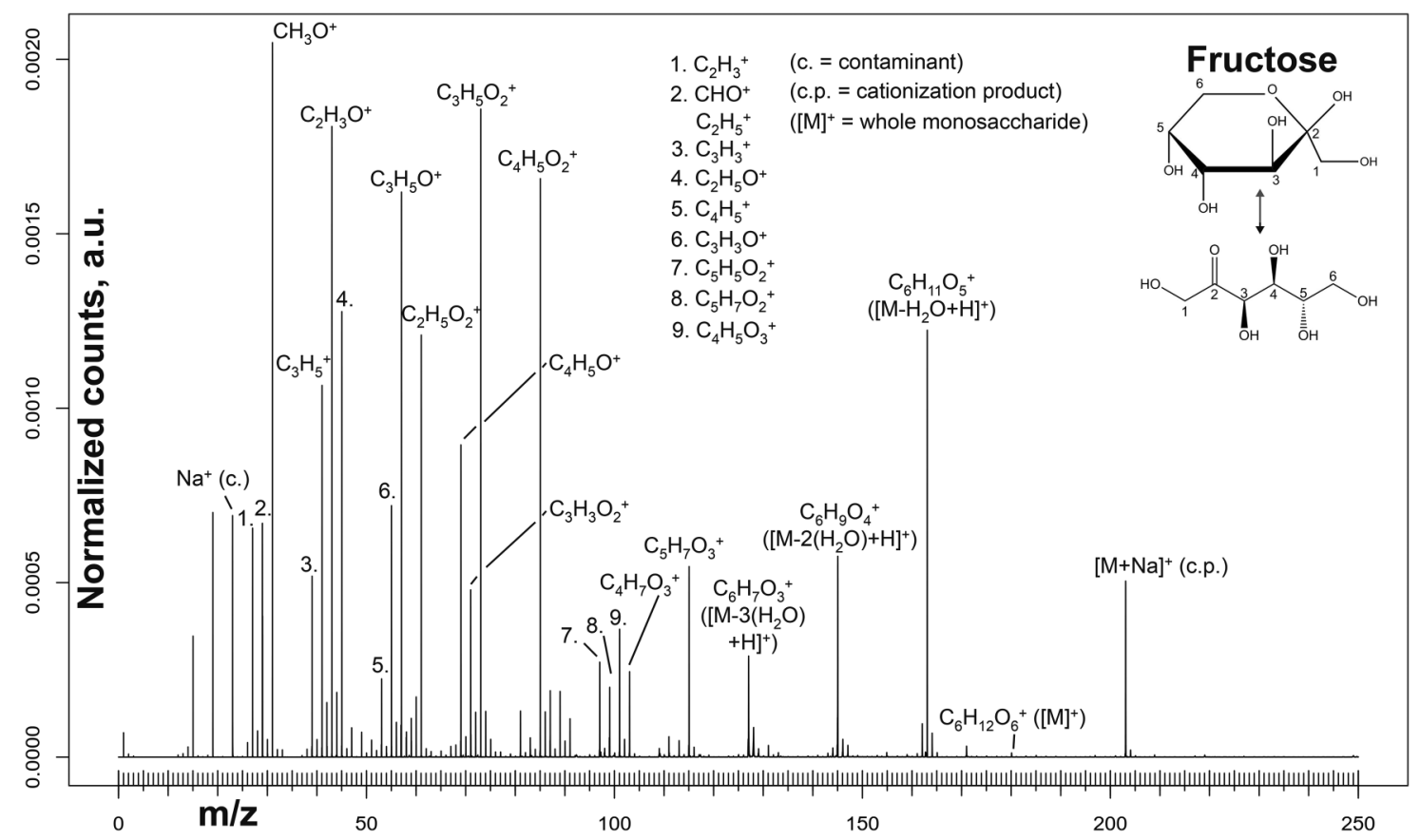

\begin{tabular}{|c|c|}
\hline Accession \#: & 01594-01 \\
\hline Host Material: & Silicon wafer \\
\hline Technique: & SIMS \\
\hline Secondary Source Polarity: & Positive \\
\hline Mass Range: & $0-250 \mathrm{Da}$ \\
\hline $\begin{array}{r}\text { Species Used for Mass } \\
\text { Calibration: }\end{array}$ & $\mathrm{CH}_{2}^{+}, \mathrm{CH}_{3}^{+}, \mathrm{C}_{2} \mathrm{H}_{4}^{+}, \mathrm{C}_{3} \mathrm{H}_{6}^{+}, \mathrm{C}_{2} \mathrm{H}_{4} \mathrm{O}^{+}$ \\
\hline Primary Ion Dose: & $9.46 \times 10^{11} \mathrm{~cm}^{-2}$ \\
\hline Primary Ion Pulse Width: & $17.2 \mathrm{~ns}$ \\
\hline Pulsed Beam Current: & $0.00074 \mathrm{nA}$ \\
\hline Biological Significance: & $\begin{array}{l}\text { D-Fructose is important for plant metabolism and energy storage, where, e.g., sucrose } \\
\text { (a.k.a. table sugar) is a disaccharide composed of one fructose and one glucose unit. } \\
\text { D-Fructose-based polysaccharides, fructans, are important storage macromolecules } \\
\text { but also function as cryoprotectants in plants regularly exposed to low temperatures } \\
\text { and reduce membrane leakage in periods drought }\end{array}$ \\
\hline
\end{tabular}




\section{D-FUCOSE}

\begin{tabular}{|c|c|c|c|}
\hline Spectrum ID \# & Mass (Da) & Species & Peak Assignment \\
\hline 01595 & 73.028 & $\mathrm{C}_{3} \mathrm{H}_{5} \mathrm{O}_{2}^{+}$ & - \\
\hline$\ldots$ & 75.044 & $\mathrm{C}_{3} \mathrm{H}_{7} \mathrm{O}_{2}^{+}$ & $\mathrm{C} 4$ to $\mathrm{C} 6$ fragment of $\mathrm{M}$ \\
\hline$\ldots$ & 85.028 & $\mathrm{C}_{4} \mathrm{H}_{5} \mathrm{O}_{2}^{+}$ & - \\
\hline$\ldots$ & 87.044 & $\mathrm{C}_{4} \mathrm{H}_{7} \mathrm{O}_{2}^{+}$ & C3 to $\mathrm{C} 6$ fragment of $\mathrm{M}-\left(\mathrm{H}_{2} \mathrm{O}\right)$ \\
\hline$\ldots$ & 105.018 & $\mathrm{C}_{3} \mathrm{H}_{5} \mathrm{O}_{4}^{+}$ & - \\
\hline$\ldots$ & 111.044 & $\mathrm{C}_{6} \mathrm{H}_{7} \mathrm{O}_{2}^{+}$ & $\mathrm{M}-3\left(\mathrm{H}_{2} \mathrm{O}\right)+\mathrm{H}$ \\
\hline$\ldots$ & 129.055 & $\mathrm{C}_{6} \mathrm{H}_{9} \mathrm{O}_{3}{ }^{+}$ & $\mathrm{M}-2\left(\mathrm{H}_{2} \mathrm{O}\right)+\mathrm{H}$ \\
\hline$\ldots$ & 147.065 & $\mathrm{C}_{6} \mathrm{H}_{11} \mathrm{O}_{4}^{+}$ & $\mathrm{M}-\left(\mathrm{H}_{2} \mathrm{O}\right)+\mathrm{H}$ \\
\hline$\ldots$ & 187.058 & {$\left[\mathrm{C}_{6} \mathrm{H}_{12} \mathrm{O}_{5}+\mathrm{Na}\right]^{+}$} & $\mathrm{M}+\mathrm{Na}$ \\
\hline
\end{tabular}

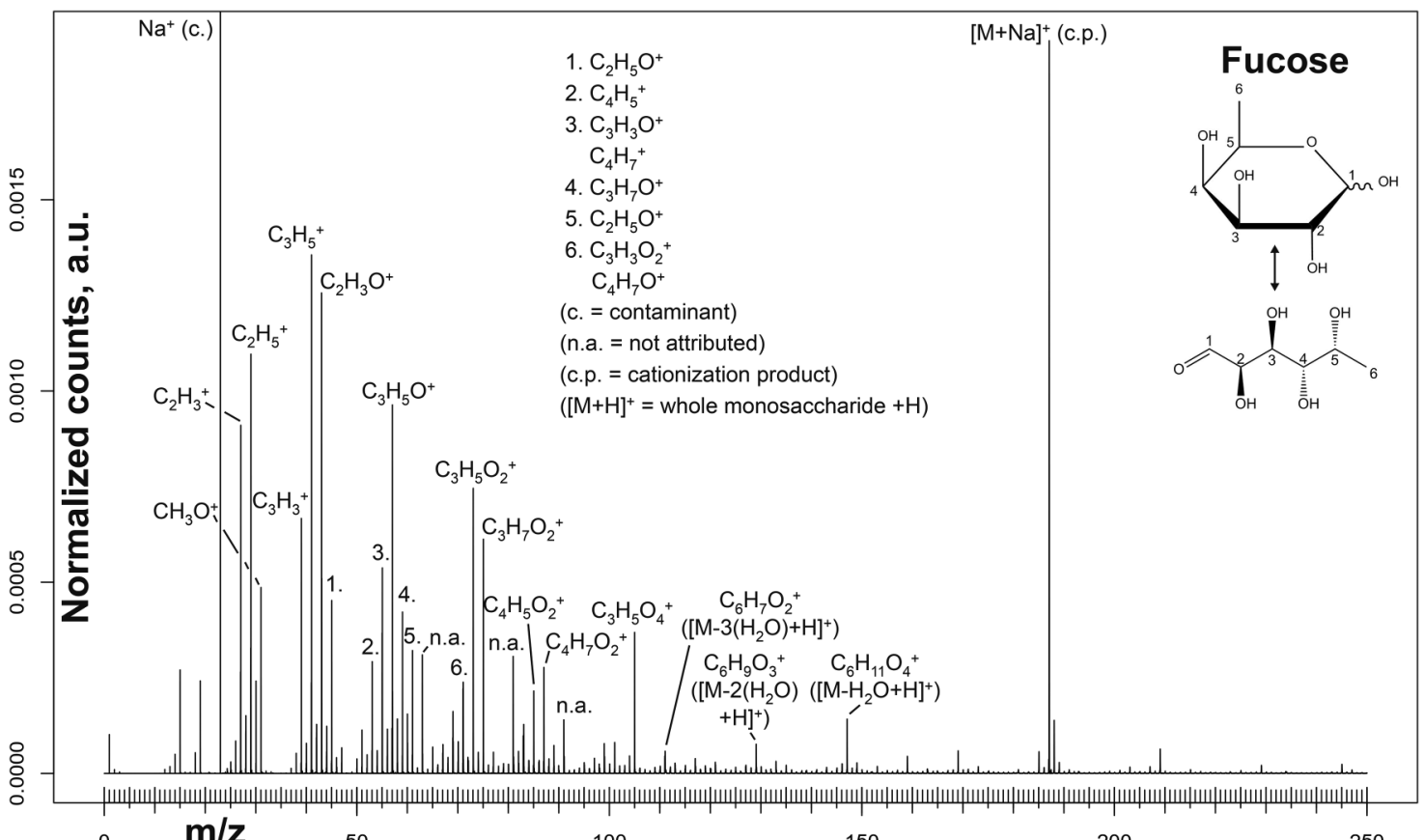

100

150

200

250

Accession \#:

01595-01

Host Material:

Technique:

Secondary Source Polarity:

Mass Range:

Species Used for Mass

Calibration:

Primary Ion Dose:

Primary Ion Pulse Width:

Pulsed Beam Current:

Biological Significance:

$$
\begin{aligned}
& \text { Silicon wafer } \\
& \text { SIMS } \\
& \text { Positive } \\
& 0-250 \mathrm{Da} \\
& \mathrm{H}_{3}{ }^{+}, \mathrm{C}_{2} \mathrm{H}_{4}^{+}, \mathrm{C}_{3} \mathrm{H}_{6}{ }^{+}, \mathrm{C}_{2} \mathrm{H}_{4} \mathrm{O}^{+} \\
& 9.71 \times 10^{11} \mathrm{~cm}^{-2} \\
& 17.2 \mathrm{~ns} \\
& 0.00076 \mathrm{nA}
\end{aligned}
$$

L-Fucose is found in a wide range of mucoproteins and many membrane glycoproteins and glycolipids (it is, e.g., part of the $\mathrm{H}$-antigen, the base unit of the membrane structure that defines the human blood type). 


\begin{tabular}{lccc}
\hline & & D-GALACTOSE \\
\hline Spectrum ID \# & Mass (Da) & Species & Peak Assignment \\
\hline 01596 & 73.028 & $\mathrm{C}_{3} \mathrm{H}_{5} \mathrm{O}_{2}{ }^{+}$ & $\mathrm{C} 4$ to $\mathrm{C} 6$ fragment of $\mathrm{M}-\left(\mathrm{H}_{2} \mathrm{O}\right)$ \\
$\ldots$ & 85.028 & $\mathrm{C}_{4} \mathrm{H}_{5} \mathrm{O}_{2}{ }^{+}$ & $\mathrm{C} 3$ to C6 fragment of $\mathrm{M}-2\left(\mathrm{H}_{2} \mathrm{O}\right)$ \\
$\ldots$ & 91.039 & $\mathrm{C}_{3} \mathrm{H}_{7} \mathrm{O}_{3}{ }^{+}$ & $\mathrm{C} 4$ to 6 fragment of $\mathrm{M}$ \\
$\ldots$ & 97.028 & $\mathrm{C}_{5} \mathrm{H}_{5} \mathrm{O}_{2}{ }^{+}$ & $\mathrm{C} 2$ to $\mathrm{C} 6$ fragment of $\mathrm{M}-3\left(\mathrm{H}_{2} \mathrm{O}\right)$ \\
$\ldots$ & 101.023 & $\mathrm{C}_{4} \mathrm{H}_{5} \mathrm{O}_{3}{ }^{+}$ & $\mathrm{C} 3$ to C6 fragment of $\mathrm{M}-\left(\mathrm{H}_{2} \mathrm{O}\right)-2 \mathrm{H}$ \\
$\ldots$ & 103.039 & $\mathrm{C}_{4} \mathrm{H}_{7} \mathrm{O}_{3}{ }^{+}$ & $\mathrm{C} 3$ to $\mathrm{C}$ fragment of $\mathrm{M}-\left(\mathrm{H}_{2} \mathrm{O}\right)$ \\
$\ldots$ & 115.039 & $\mathrm{C}_{5} \mathrm{H}_{7} \mathrm{O}_{3}{ }^{+}$ & $\mathrm{C} 2$ to $\mathrm{C} 6$ fragment of $\mathrm{M}-2\left(\mathrm{H}_{2} \mathrm{O}\right)$ \\
$\ldots$ & 127.039 & $\mathrm{C}_{6} \mathrm{H}_{7} \mathrm{O}_{3}{ }^{+}$ & $\mathrm{M}-3\left(\mathrm{H}_{2} \mathrm{O}\right)+\mathrm{H}$ \\
$\ldots$ & 145.050 & $\mathrm{C}_{6} \mathrm{H}_{9} \mathrm{O}_{4}{ }^{+}$ & $\mathrm{M}-2\left(\mathrm{H}_{2} \mathrm{O}\right)+\mathrm{H}$ \\
\hline & 163.060 & $\mathrm{C}_{6} \mathrm{H}_{11} \mathrm{O}_{5}{ }^{+}$ & $\mathrm{M}-\left(\mathrm{H}_{2} \mathrm{O}\right)+\mathrm{H}$ \\
\hline
\end{tabular}

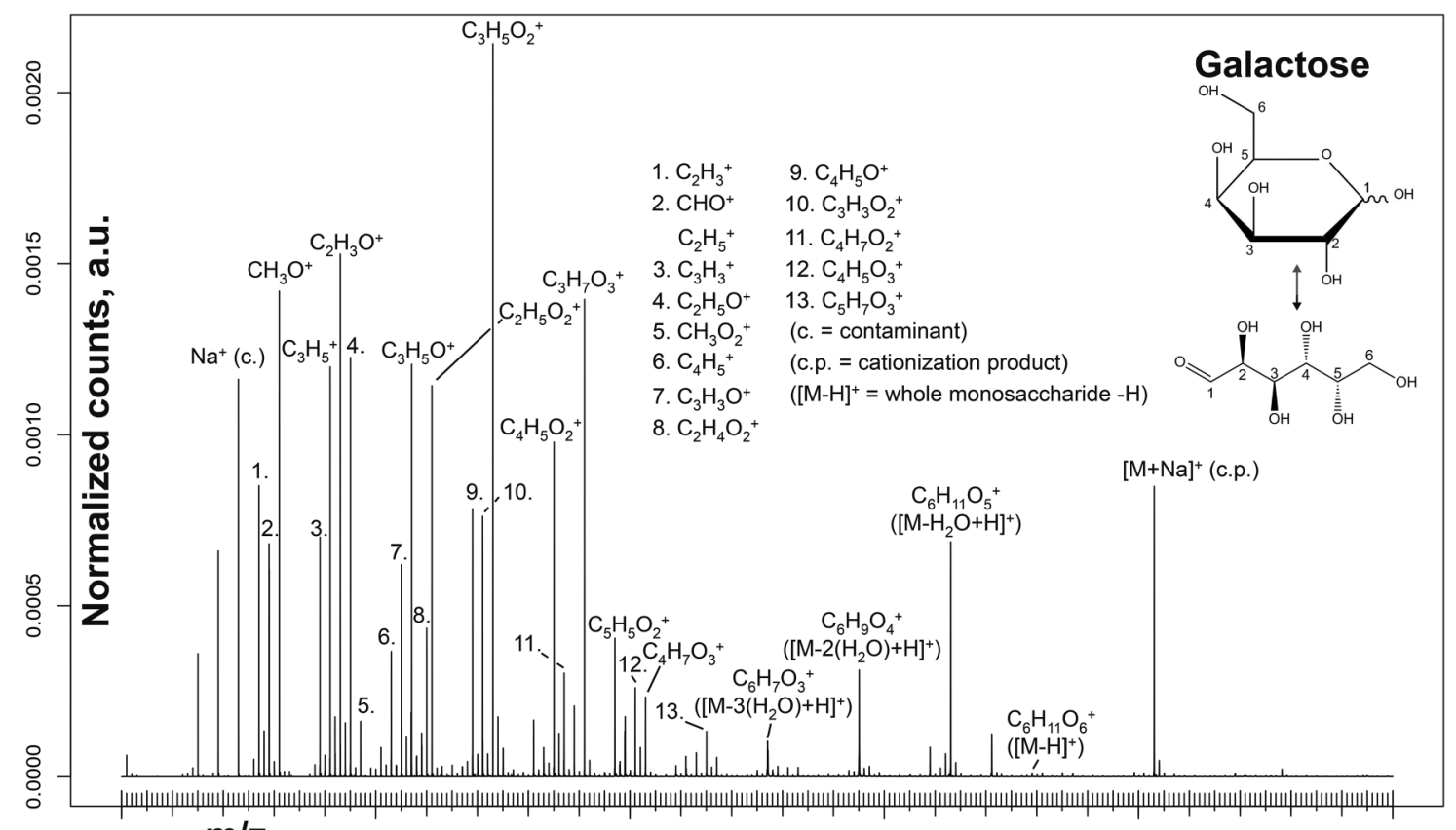

o $\mathrm{m} / \mathbf{z}$

50

100

150

200

250

\begin{tabular}{rc}
\hline Accession \#: & $01596-01$ \\
\hline Host Material: & Silicon wafer \\
Technique: & SIMS \\
Secondary Source Polarity: \\
Mass Range: & Positive \\
Species Used for Mass & $0-250 \mathrm{Da}$ \\
Calibration: & $\mathrm{CH}_{2}{ }^{+}, \mathrm{CH}_{3}{ }^{+}, \mathrm{C}_{2} \mathrm{H}_{4}{ }^{+}, \mathrm{C}_{3} \mathrm{H}_{6}{ }^{+}, \mathrm{C}_{2} \mathrm{H}_{4} \mathrm{O}^{+}$ \\
Primary lon Dose: & $9.71 \times 10^{11} \mathrm{~cm}^{-2}$ \\
Primary lon Pulse Width: & $17.2 \mathrm{~ns}^{-2}$ \\
Pulsed Beam Current: & $0.00076 \mathrm{nA}$ \\
Biological Significance: & D-Galactose is found in a wide range of glycoproteins and glycolipids and \\
& galactose-based polysaccharides occur in certain types of hemicellulose. The milk \\
& sugar lactose is a disaccharide composed of one D-galactose and one D-glucose unit \\
\hline
\end{tabular}




\begin{tabular}{|c|c|c|c|}
\hline \multicolumn{4}{|c|}{ 2-DEOXY-D-GALACTOSE } \\
\hline Spectrum ID \# & Mass (Da) & Species & Peak Assignment \\
\hline 01597 & 69.033 & $\mathrm{C}_{4} \mathrm{H}_{5} \mathrm{O}^{+}$ & - \\
\hline$\ldots$ & 73.028 & $\mathrm{C}_{3} \mathrm{H}_{5} \mathrm{O}_{2}^{+}$ & $\mathrm{C} 4$ to $\mathrm{C} 6$ fragment of $\mathrm{M}-\left(\mathrm{H}_{2} \mathrm{O}\right)$ \\
\hline$\ldots$ & 83.049 & $\mathrm{C}_{5} \mathrm{H}_{7} \mathrm{O}^{+}$ & - \\
\hline$\ldots$ & 85.028 & $\mathrm{C}_{4} \mathrm{H}_{5} \mathrm{O}_{2}^{+}$ & $\mathrm{C} 3$ to $\mathrm{C} 6$ fragment of $\mathrm{M}-2\left(\mathrm{H}_{2} \mathrm{O}\right)$ \\
\hline$\ldots$ & 97.028 & $\mathrm{C}_{5} \mathrm{H}_{5} \mathrm{O}_{2}^{+}$ & $\mathrm{C} 2$ to $\mathrm{C} 6$ fragment of $\mathrm{M}-2\left(\mathrm{H}_{2} \mathrm{O}\right)-2 \mathrm{H}$ \\
\hline$\ldots$ & 99.044 & $\mathrm{C}_{5} \mathrm{H}_{7} \mathrm{O}_{2}^{+}$ & $\mathrm{C} 2$ to $\mathrm{C} 6$ fragment of $\mathrm{M}-2\left(\mathrm{H}_{2} \mathrm{O}\right)$ \\
\hline$\ldots$ & 103.039 & $\mathrm{C}_{4} \mathrm{H}_{7} \mathrm{O}_{3}^{+}$ & $\mathrm{C} 3$ to $\mathrm{C} 6$ fragment of $\mathrm{M}-\left(\mathrm{H}_{2} \mathrm{O}\right)$ \\
\hline$\ldots$ & 111.044 & $\mathrm{C}_{6} \mathrm{H}_{7} \mathrm{O}_{2}^{+}$ & $\mathrm{M}-3\left(\mathrm{H}_{2} \mathrm{O}\right)+\mathrm{H}$ \\
\hline$\ldots$ & 125.055 & $\mathrm{C}_{6} \mathrm{H}_{9} \mathrm{O}_{3}^{+}$ & $\mathrm{M}-2\left(\mathrm{H}_{2} \mathrm{O}\right)+\mathrm{H}$ \\
\hline$\ldots$ & 147.065 & $\mathrm{C}_{6} \mathrm{H}_{11} \mathrm{O}_{4}^{+}$ & $\mathrm{M}-\left(\mathrm{H}_{2} \mathrm{O}\right)+\mathrm{H}$ \\
\hline
\end{tabular}

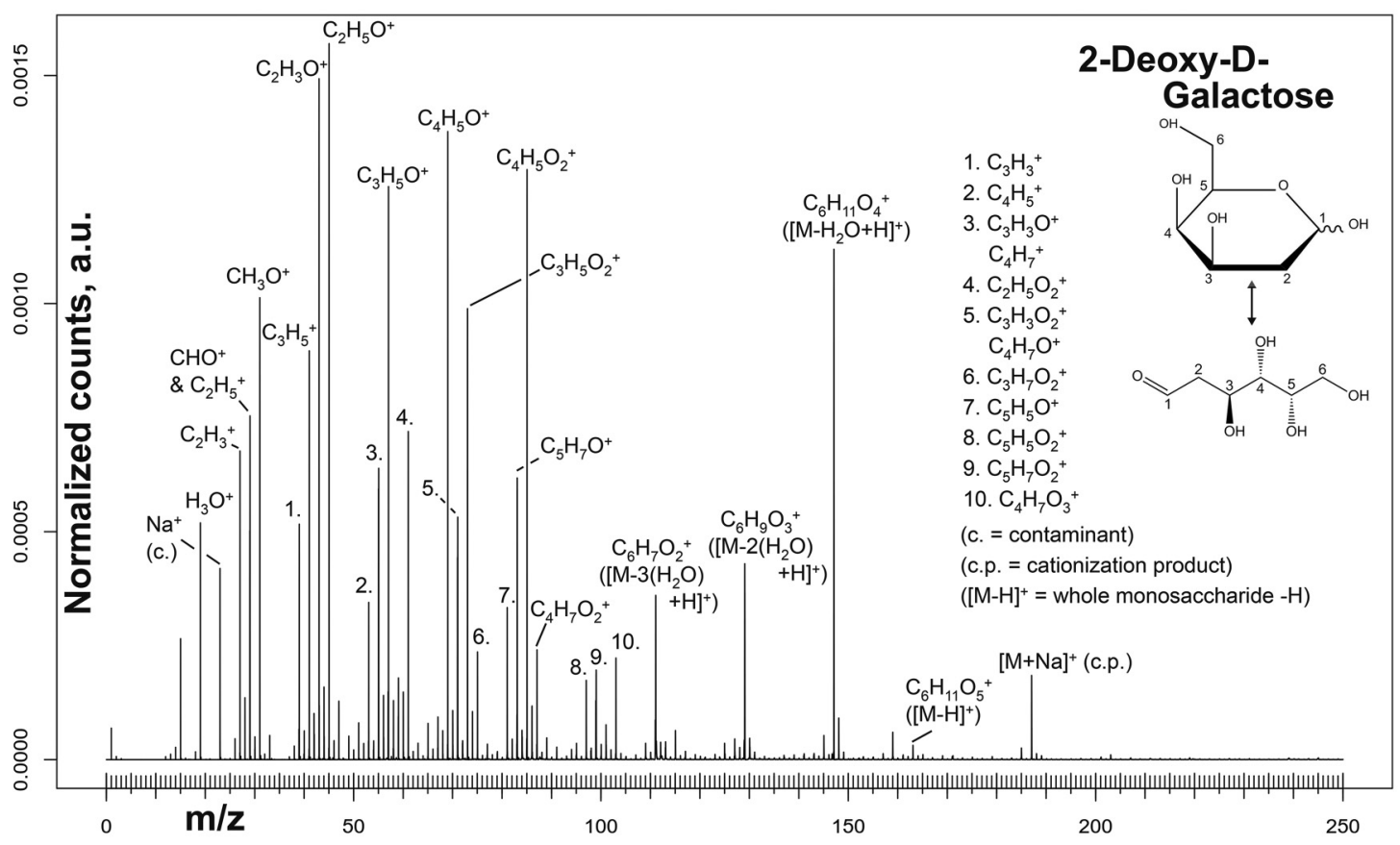

\begin{tabular}{rc}
\hline Accession \#: & $01597-01$ \\
\hline Host Material: & Silicon wafer \\
$\mathbf{\square}$ Technique: & $\mathrm{SIMS}$ \\
Mecondary Source Polarity: & Positive \\
Mass Range: & $0-250 \mathrm{Da}$ \\
Species Used for Mass Calibration: & $\mathrm{CH}_{2}^{+}, \mathrm{CH}_{3}^{+}, \mathrm{C}_{2} \mathrm{H}_{4}^{+}, \mathrm{C}_{3} \mathrm{H}_{6}^{+}, \mathrm{C}_{2} \mathrm{H}_{4} \mathrm{O}^{+}$ \\
Primary lon Dose: & $9.71 \times 10^{11} \mathrm{~cm}^{-2}$ \\
Primary lon Pulse Width: & $17.2 \mathrm{~ns}$ \\
Pulsed Beam Current: & $0.00076 \mathrm{nA}$ \\
Biological Significance: & 2-Deoxy-D-galactose is not abundant in nature but of scientific \\
& relevance as a known inhibitor of membrane glycoprotein synthesis \\
\hline
\end{tabular}




\section{D-GALACTURONIC ACID}

\begin{tabular}{lccc}
\hline Spectrum ID \# & Mass (Da) & Species & Peak Assignment \\
\hline 01598 & 71.013 & $\mathrm{C}_{3} \mathrm{H}_{3} \mathrm{O}_{2}{ }^{+}$ & - \\
$\ldots$ & 73.028 & $\mathrm{C}_{3} \mathrm{H}_{5} \mathrm{O}_{2}{ }^{+}$ & - \\
$\ldots$ & 85.028 & $\mathrm{C}_{4} \mathrm{H}_{5} \mathrm{O}_{2}{ }^{+}$ & - \\
$\ldots$ & 89.023 & $\mathrm{C}_{3} \mathrm{H}_{5} \mathrm{O}_{3}{ }^{+}$ & $\mathrm{C} 1$ to $\mathrm{C} 3$ fragment of $\mathrm{M}+\mathrm{H}$ \\
$\ldots$ & 97.028 & $\mathrm{C}_{5} \mathrm{H}_{5} \mathrm{O}_{2}{ }^{+}$ & - \\
$\ldots$ & 101.023 & $\mathrm{C}_{4} \mathrm{H}_{5} \mathrm{O}_{3}{ }^{+}$ & $\mathrm{C} 1$ to $\mathrm{C}$ fragment of $\mathrm{M}-\left(\mathrm{H}_{2} \mathrm{O}\right)+\mathrm{H}$ \\
$\ldots$ & 113.023 & $\mathrm{C}_{5} \mathrm{H}_{5} \mathrm{O}_{3}{ }^{+}$ & $\mathrm{C} 1$ to $\mathrm{C} 5$ fragment of $\mathrm{M}-2\left(\mathrm{H}_{2} \mathrm{O}\right)$ \\
$\ldots$ & 131.034 & $\mathrm{C}_{5} \mathrm{H}_{7} \mathrm{O}_{4}{ }^{+}$ & $\mathrm{C} 1$ to $\mathrm{C} 5$ fragment of $\mathrm{M}-\left(\mathrm{H}_{2} \mathrm{O}\right)$ \\
$\ldots$ & 141.018 & $\mathrm{C}_{6} \mathrm{H}_{5} \mathrm{O}_{4}{ }^{+}$ & $\mathrm{M}-3\left(\mathrm{H}_{2} \mathrm{O}\right)+\mathrm{H}$ \\
$\ldots$ & 159.029 & $\mathrm{C}_{6} \mathrm{H}_{7} \mathrm{O}_{5}{ }^{+}$ & $\mathrm{M}-2\left(\mathrm{H}_{2} \mathrm{O}\right)+\mathrm{H}$ \\
$\ldots$ & 177.039 & $\mathrm{C}_{6} \mathrm{H}_{9} \mathrm{O}_{6}{ }^{+}$ & $\mathrm{M}-\left(\mathrm{H}_{2} \mathrm{O}\right)+\mathrm{H}$ \\
$\ldots$ & 212.076 & {$\left[\mathrm{C}_{6} \mathrm{H}_{10} \mathrm{O}_{7}+\mathrm{NH}_{4}\right]^{+}$} & $\mathrm{M}+\mathrm{NH}_{4}$ \\
\hline
\end{tabular}

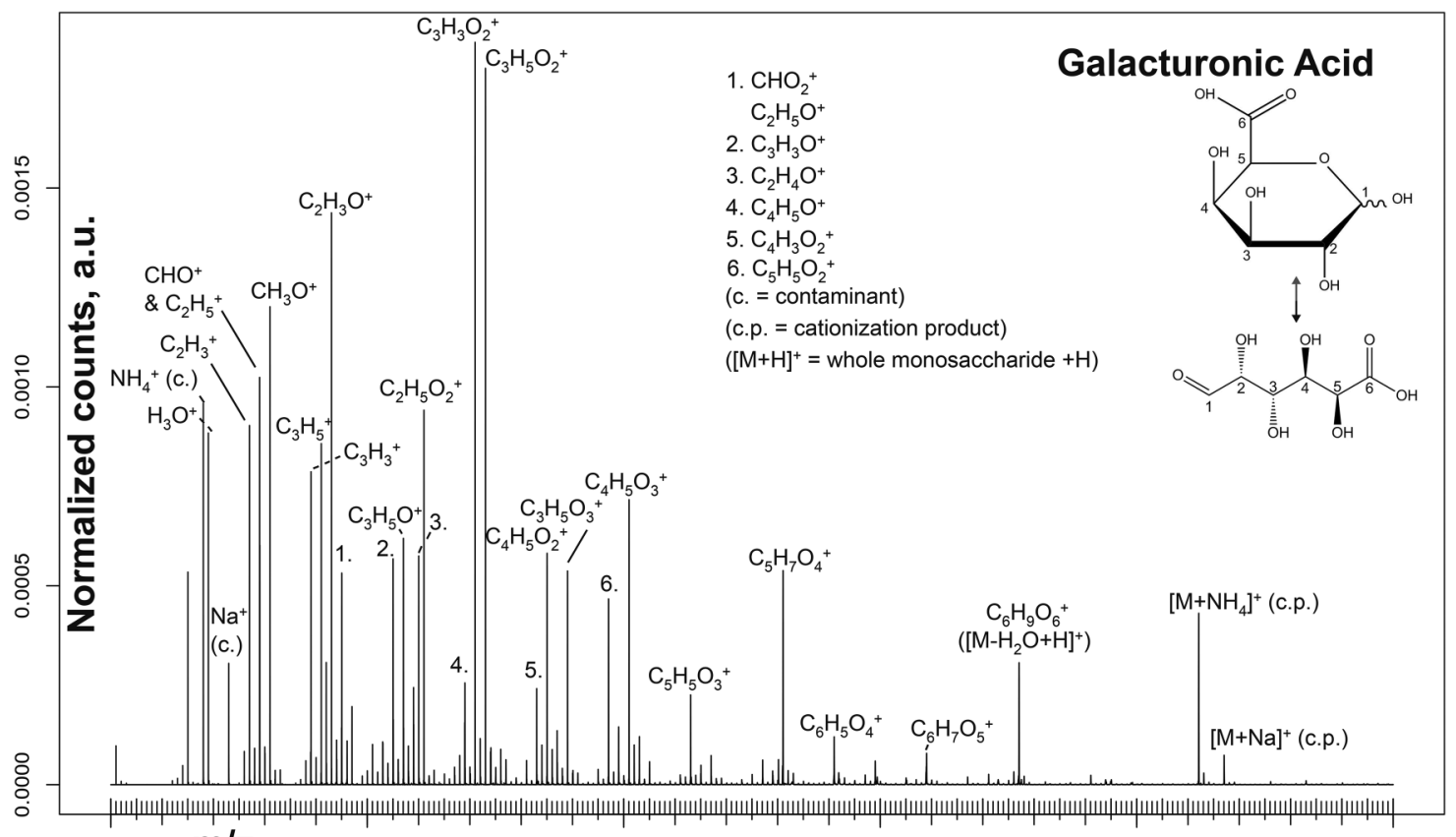
o $\quad \mathbf{m} / \mathbf{z}$
50
100
150
200
250

\begin{tabular}{rc}
\hline Accession \#: & $01598-01$ \\
\hline Host Material: & Silicon wafer \\
Technique: & SIMS \\
Mass Range: & Positive \\
Secondary Source Polarity: & $0-250 \mathrm{Da}$ \\
Species Used for Mass Calibration: & $\mathrm{CH}_{2}^{+}, \mathrm{CH}_{3}^{+}, \mathrm{C}_{2} \mathrm{H}_{4}^{+}, \mathrm{C}_{3} \mathrm{H}_{6}{ }^{+}, \mathrm{C}_{2} \mathrm{H}_{4} \mathrm{O}^{+}$ \\
Primary lon Dose: & $9.71 \times 10^{11} \mathrm{~cm}^{-2}$ \\
Primary lon Pulse Width: & $17.2 \mathrm{~ns}$ \\
Pulsed Beam Current: & $0.00076 \mathrm{nA}$ \\
Biological Significance: & D-Galacturonic acid is the uronic acid of D-galactose and is, among others, \\
& the main component of the plant cell wall heteropolysaccharide pectin \\
\hline
\end{tabular}




\begin{tabular}{|c|c|c|c|}
\hline \multicolumn{4}{|c|}{ D-GLUCOSE } \\
\hline Spectrum ID \# & Mass (Da) & Species & Peak Assignment \\
\hline 01599 & 73.028 & $\mathrm{C}_{3} \mathrm{H}_{5} \mathrm{O}_{2}^{+}$ & $\mathrm{C} 4$ to $\mathrm{C} 6$ fragment of $\mathrm{M}-\left(\mathrm{H}_{2} \mathrm{O}\right)$ \\
\hline$\ldots$ & 85.028 & $\mathrm{C}_{4} \mathrm{H}_{5} \mathrm{O}_{2}^{+}$ & C3 to $\mathrm{C} 6$ fragment of $\mathrm{M}-2\left(\mathrm{H}_{2} \mathrm{O}\right)$ \\
\hline$\ldots$ & 87.044 & $\mathrm{C}_{4} \mathrm{H}_{7} \mathrm{O}_{2}^{+}$ & $\mathrm{C} 3$ to $\mathrm{C} 6$ fragment of $\mathrm{M}-2\left(\mathrm{H}_{2} \mathrm{O}\right)+2 \mathrm{H}$ \\
\hline$\ldots$ & 91.039 & $\mathrm{C}_{3} \mathrm{H}_{7} \mathrm{O}_{3}^{+}$ & $\mathrm{C} 4$ to $\mathrm{C} 6$ fragment of $\mathrm{M}$ \\
\hline$\ldots$ & 97.028 & $\mathrm{C}_{5} \mathrm{H}_{5} \mathrm{O}_{2}^{+}$ & $\mathrm{C} 2$ to $\mathrm{C} 6$ fragment of $\mathrm{M}-3\left(\mathrm{H}_{2} \mathrm{O}\right)$ \\
\hline$\ldots$ & 103.039 & $\mathrm{C}_{4} \mathrm{H}_{7} \mathrm{O}_{3}^{+}$ & C3 to $\mathrm{C} 6$ fragment of $\mathrm{M}-\left(\mathrm{H}_{2} \mathrm{O}\right)$ \\
\hline$\ldots$ & 115.039 & $\mathrm{C}_{5} \mathrm{H}_{7} \mathrm{O}_{3}^{+}$ & $\mathrm{C} 2$ to $\mathrm{C} 6$ fragment of $\mathrm{M}-2\left(\mathrm{H}_{2} \mathrm{O}\right)$ \\
\hline$\ldots$ & 127.039 & $\mathrm{C}_{6} \mathrm{H}_{7} \mathrm{O}_{3}^{+}$ & $\mathrm{M}-3\left(\mathrm{H}_{2} \mathrm{O}\right)+\mathrm{H}$ \\
\hline$\ldots$ & 145.050 & $\mathrm{C}_{6} \mathrm{H}_{9} \mathrm{O}_{4}^{+}$ & $\mathrm{M}-2\left(\mathrm{H}_{2} \mathrm{O}\right)+\mathrm{H}$ \\
\hline$\ldots$ & 163.060 & $\mathrm{C}_{6} \mathrm{H}_{11} \mathrm{O}_{5}^{+}$ & $\mathrm{M}-\left(\mathrm{H}_{2} \mathrm{O}\right)+\mathrm{H}$ \\
\hline
\end{tabular}

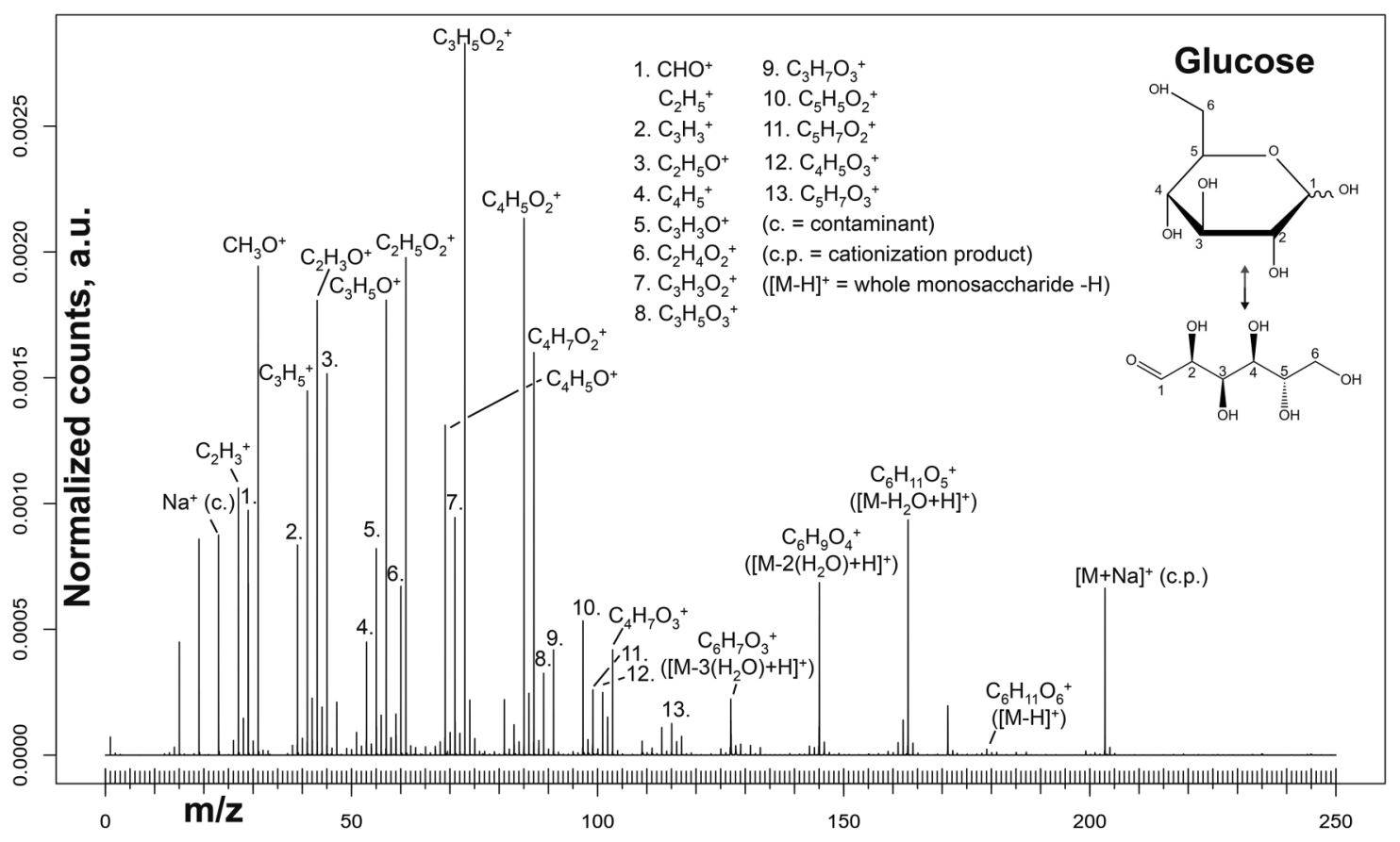

\begin{tabular}{|c|c|}
\hline Accession \#: & 01599-01 \\
\hline Dost Material: & Silicon wafer \\
\hline Technique: & SIMS \\
\hline Secondary Source Polarity: & Positive \\
\hline Mass Range: & $0-250 \mathrm{Da}$ \\
\hline Species Used for Mass Calibration: & $\mathrm{CH}_{2}^{+}, \mathrm{CH}_{3}^{+}, \mathrm{C}_{2} \mathrm{H}_{4}^{+}, \mathrm{C}_{3} \mathrm{H}_{6}^{+}, \mathrm{C}_{2} \mathrm{H}_{4} \mathrm{O}^{+}$ \\
\hline Primary Ion Dose: & $9.71 \times 10^{12} \mathrm{~cm}^{-2}$ \\
\hline Primary Ion Pulse Width: & $17.2 \mathrm{~ns}$ \\
\hline Pulsed Beam Current: & $0.00076 n A$ \\
\hline Biological Significance: & $\begin{array}{l}\text { Glucose is essential for energy storage, energy transport, and cell metabolism in } \\
\text { animals, plants, and microorganisms. Glucose is the monomer unit of the } \\
\text { polysaccharides starch and glycogen, polymers acting as energy storage in } \\
\text { plants and animals, respectively, and the polysaccharide cellulose, which is the } \\
\text { main plant cell wall component. Glucose is also part of many di- and } \\
\text { oligosaccharides, e.g., sucrose, lactose, and trehalose }\end{array}$ \\
\hline
\end{tabular}




\begin{tabular}{lccc}
\hline & & D-GLUCURONIC ACID & \\
\hline Spectrum ID \# & Mass (Da) & Species & Peak Assignment \\
\hline 01600 & 71.013 & $\mathrm{C}_{3} \mathrm{H}_{3} \mathrm{O}_{2}{ }^{+}$ & - \\
$\ldots$ & 73.028 & $\mathrm{C}_{3} \mathrm{H}_{5} \mathrm{O}_{2}{ }^{+}$ & - \\
$\ldots$ & 85.028 & $\mathrm{C}_{4} \mathrm{H}_{5} \mathrm{O}_{2}{ }^{+}$ & - \\
$\ldots$ & 89.023 & $\mathrm{C}_{3} \mathrm{H}_{5} \mathrm{O}_{3}{ }^{+}$ & $\mathrm{C} 1$ to $\mathrm{C} 3$ fragment of $\mathrm{M}+\mathrm{H}$ \\
$\ldots$ & 97.028 & $\mathrm{C}_{5} \mathrm{H}_{5} \mathrm{O}_{2}{ }^{+}$ & - \\
$\ldots$ & 99.008 & $\mathrm{C}_{4} \mathrm{H}_{3} \mathrm{O}_{3}{ }^{+}$ & $\mathrm{C} 1$ to $\mathrm{C} 4$ fragment of $\mathrm{M}-\left(\mathrm{H}_{2} \mathrm{O}\right)-\mathrm{H}$ \\
$\ldots$ & 101.023 & $\mathrm{C}_{4} \mathrm{H}_{5} \mathrm{O}_{3}{ }^{+}$ & $\mathrm{C} 1$ to $\mathrm{C} 4$ fragment of M $-\left(\mathrm{H}_{2} \mathrm{O}\right)+\mathrm{H}$ \\
$\ldots$ & 113.023 & $\mathrm{C}_{5} \mathrm{H}_{5} \mathrm{O}_{3}{ }^{+}$ & $\mathrm{C} 1$ to $\mathrm{C} 5$ fragment of M $-2\left(\mathrm{H}_{2} \mathrm{O}\right)$ \\
$\ldots$ & 117.055 & $\mathrm{C}_{5} \mathrm{H}_{9} \mathrm{O}_{3}{ }^{+}$ & - \\
$\ldots$ & 131.034 & $\mathrm{C}_{5} \mathrm{H}_{7} \mathrm{O}_{4}{ }^{+}$ & $\mathrm{C} 1$ to $\mathrm{C} 5$ fragment of M $-\left(\mathrm{H}_{2} \mathrm{O}\right)$ \\
$\ldots$ & 141.018 & $\mathrm{C}_{6} \mathrm{H}_{5} \mathrm{O}_{4}{ }^{+}$ & $\mathrm{M}-3\left(\mathrm{H}_{2} \mathrm{O}\right)+\mathrm{H}$ \\
$\ldots$ & 159.029 & $\mathrm{C}_{6} \mathrm{H}_{7} \mathrm{O}_{5}{ }^{+}$ & $\mathrm{M}-2\left(\mathrm{H}_{2} \mathrm{O}\right)+\mathrm{H}$ \\
$\ldots$ & 177.039 & $\mathrm{C}_{6} \mathrm{H}_{9} \mathrm{O}_{6}{ }^{+}$ & $\mathrm{M}-\left(\mathrm{H}_{2} \mathrm{O}\right)+\mathrm{H}$ \\
\hline & 194.042 & $\mathrm{C}_{6} \mathrm{H}_{10} \mathrm{O}_{7}{ }^{+}$ & $\mathrm{M}$ \\
\hline
\end{tabular}

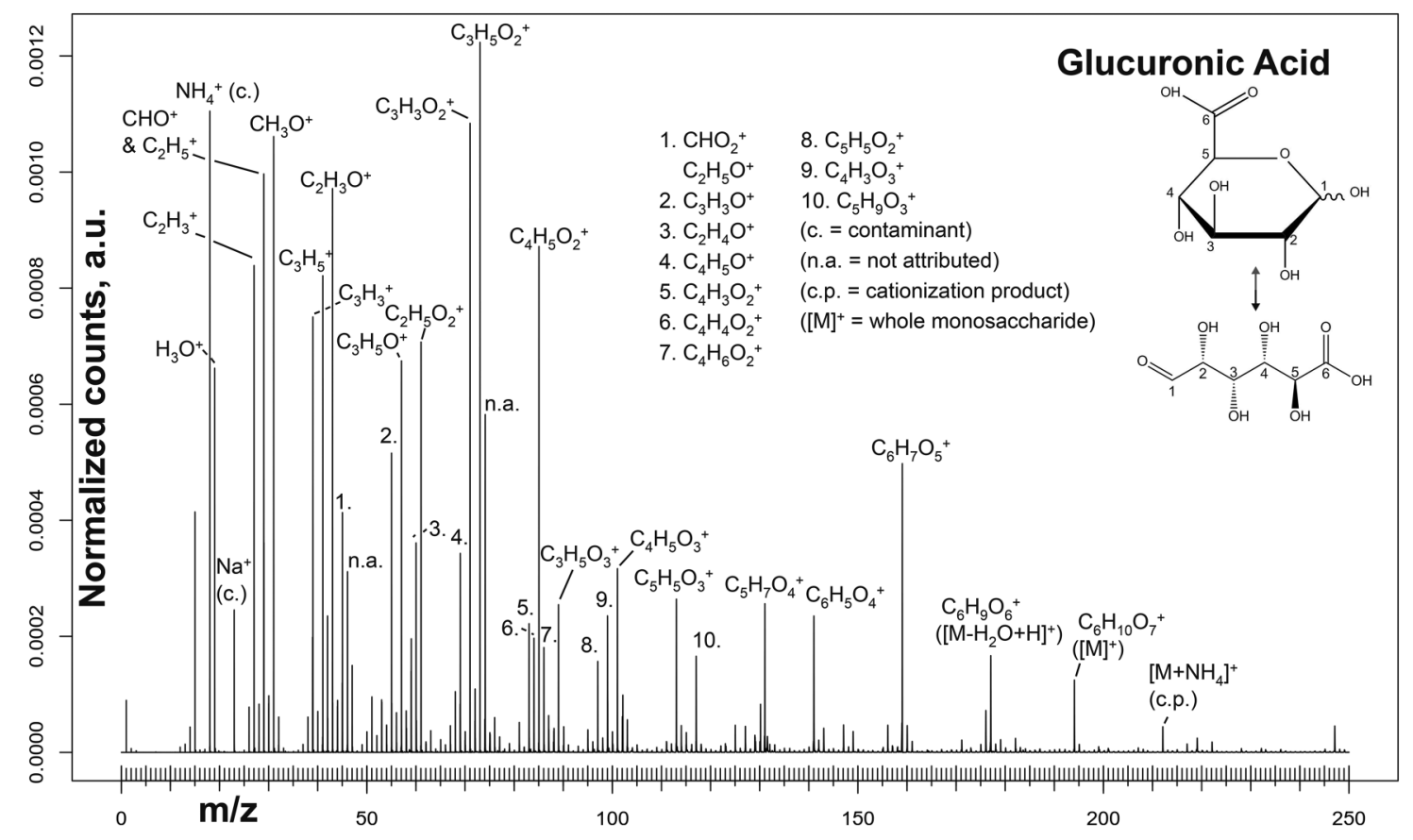

\begin{tabular}{rc}
\hline Accession \#: & $01600-01$ \\
\hline Host Material: & Silicon wafer \\
Technique: & SIMS \\
Secondary Source Polarity: & Positive \\
Mass Range: & $0-250 \mathrm{Da}$ \\
Species Used for Mass Calibration: & $\mathrm{CH}_{2}{ }^{+}, \mathrm{CH}_{3}^{+}, \mathrm{C}_{2} \mathrm{H}_{4}^{+}, \mathrm{C}_{3} \mathrm{H}_{6}{ }^{+}, \mathrm{C}_{2} \mathrm{H}_{4} \mathrm{O}^{+}$ \\
Primary lon Dose: & $9.71 \times 10^{11} \mathrm{~cm}^{-2}$ \\
Primary lon Pulse Width: & $17.2 \mathrm{~ns}$ \\
Pulsed Beam Current: & $0.00076 \mathrm{nA}$ \\
Biological Significance: & Glucuronic acid is the uronic acid of D-glucose and plays a major role in the \\
& phase Il detoxification processes in the liver, where in an enzyme-driven \\
& reaction it is bound to toxins to facilitate their excretion \\
\hline
\end{tabular}




\begin{tabular}{|c|c|c|c|}
\hline \multicolumn{4}{|c|}{ D-MANNOSE } \\
\hline Spectrum ID \# & Mass (Da) & Species & Peak Assignment \\
\hline 01601 & 73.028 & $\mathrm{C}_{3} \mathrm{H}_{5} \mathrm{O}_{2}^{+}$ & $\mathrm{C} 4$ to $\mathrm{C} 6$ fragment of $\mathrm{M}-\left(\mathrm{H}_{2} \mathrm{O}\right)$ \\
\hline$\ldots$ & 85.028 & $\mathrm{C}_{4} \mathrm{H}_{5} \mathrm{O}_{2}^{+}$ & C3 to $\mathrm{C} 6$ fragment of $\mathrm{M}-2\left(\mathrm{H}_{2} \mathrm{O}\right)$ \\
\hline$\ldots$ & 87.044 & $\mathrm{C}_{4} \mathrm{H}_{7} \mathrm{O}_{2}^{+}$ & $\mathrm{C} 3$ to $\mathrm{C} 6$ fragment of $\mathrm{M}-2\left(\mathrm{H}_{2} \mathrm{O}\right)+2 \mathrm{H}$ \\
\hline$\ldots$ & 91.039 & $\mathrm{C}_{3} \mathrm{H}_{7} \mathrm{O}_{3}^{+}$ & C4 to $\mathrm{C} 6$ fragment of $\mathrm{M}$ \\
\hline$\ldots$ & 97.028 & $\mathrm{C}_{5} \mathrm{H}_{5} \mathrm{O}_{2}^{+}$ & $\mathrm{C} 2$ to $\mathrm{C} 6$ fragment of $\mathrm{M}-3\left(\mathrm{H}_{2} \mathrm{O}\right)$ \\
\hline$\ldots$ & 103.039 & $\mathrm{C}_{4} \mathrm{H}_{7} \mathrm{O}_{3}^{+}$ & C3 to $\mathrm{C} 6$ fragment of $\mathrm{M}-\left(\mathrm{H}_{2} \mathrm{O}\right)$ \\
\hline$\ldots$ & 115.039 & $\mathrm{C}_{5} \mathrm{H}_{7} \mathrm{O}_{3}^{+}$ & $\mathrm{C} 2$ to $\mathrm{C} 6$ fragment of $\mathrm{M}-2\left(\mathrm{H}_{2} \mathrm{O}\right)$ \\
\hline$\ldots$ & 127.039 & $\mathrm{C}_{6} \mathrm{H}_{7} \mathrm{O}_{3}^{+}$ & $\mathrm{M}-3\left(\mathrm{H}_{2} \mathrm{O}\right)+\mathrm{H}$ \\
\hline$\ldots$ & 145.050 & $\mathrm{C}_{6} \mathrm{H}_{9} \mathrm{O}_{4}^{+}$ & $\mathrm{M}-2\left(\mathrm{H}_{2} \mathrm{O}\right)+\mathrm{H}$ \\
\hline$\ldots$ & 163.060 & $\mathrm{C}_{6} \mathrm{H}_{11} \mathrm{O}_{5}^{+}$ & $\mathrm{M}-\left(\mathrm{H}_{2} \mathrm{O}\right)+\mathrm{H}$ \\
\hline
\end{tabular}

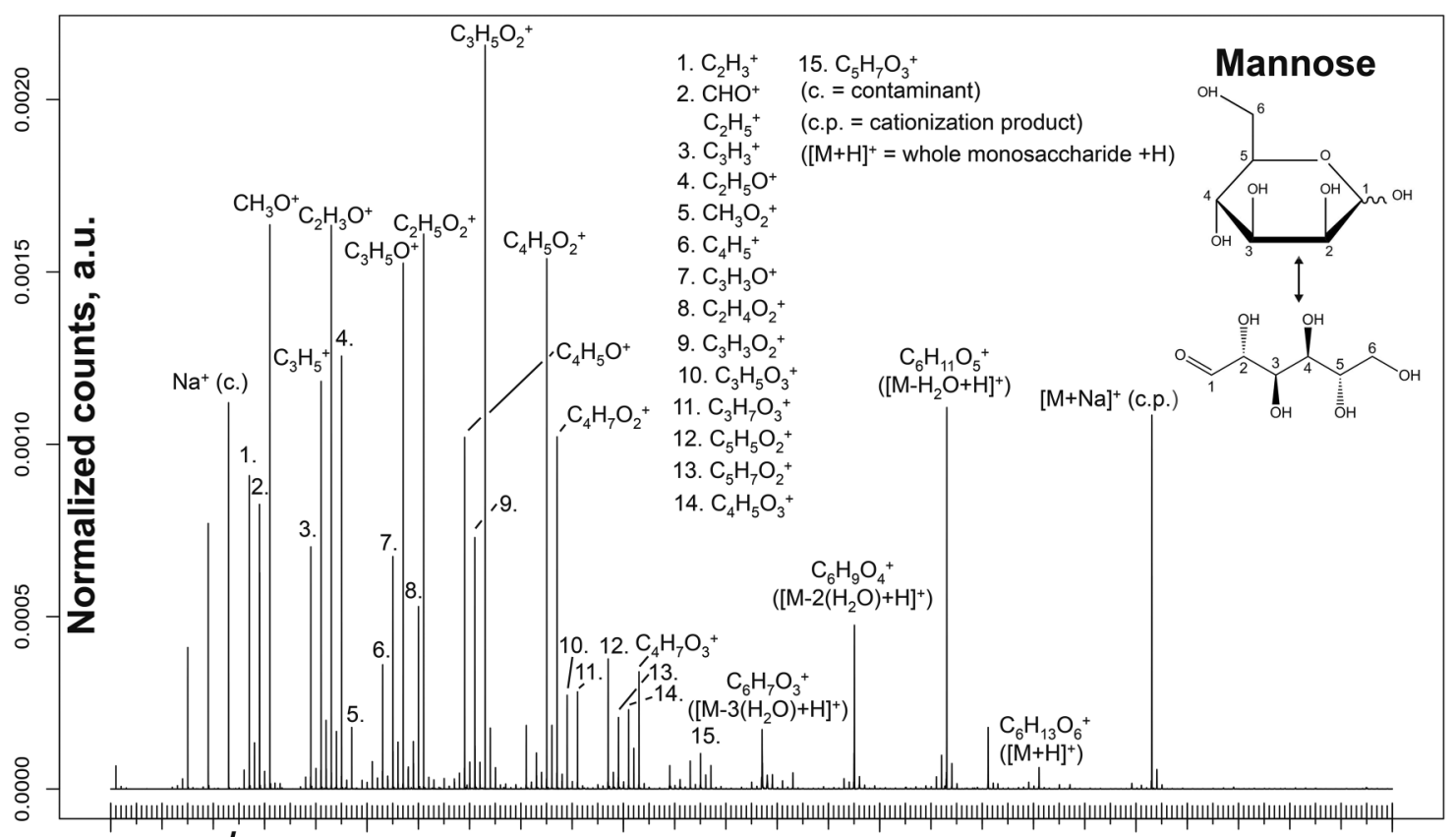

$\begin{array}{llllll}0 & \mathbf{m} / \mathbf{Z} & 50 & 100 & 150 & 200\end{array}$

\begin{tabular}{rc}
\hline Accession \#: & $01601-01$ \\
\hline Host Material: & Silicon wafer \\
Technique: & SIMS \\
Mass Range: & Positive \\
Secondary Source Polarity: & $0-250 \mathrm{Da}$ \\
Species Used for Mass Calibration: & $\mathrm{CH}_{2}{ }^{+}, \mathrm{CH}_{3}^{+}, \mathrm{C}_{2} \mathrm{H}_{4}^{+}, \mathrm{C}_{3} \mathrm{H}_{6}{ }^{+}, \mathrm{C}_{2} \mathrm{H}_{4} \mathrm{O}^{+}$ \\
Primary lon Dose: & $9.71 \times 10^{11} \mathrm{~cm}^{-2}$ \\
Primary lon Pulse Width: & $17.2 \mathrm{~ns}$ \\
Pulsed Beam Current: & $0.00076 \mathrm{nA}$ \\
Biological Significance: & D-Mannose is part of the glycosylation process of certain glycoproteins. \\
& During the C-mannosylation process, a mannose monosaccharide \\
& attaches by covalent bond formation to tryptophan \\
\hline
\end{tabular}




\begin{tabular}{lccc}
\hline & & MYO-INOSITOL & \\
\hline Spectrum ID \# & Mass(Da) & Species & Peak Assignment \\
\hline 01602 & 73.028 & $\mathrm{C}_{3} \mathrm{H}_{5} \mathrm{O}_{2}{ }^{+}$ & $\mathrm{C} 4$ to $\mathrm{C} 6$ fragment of $\mathrm{M}-\left(\mathrm{H}_{2} \mathrm{O}\right)$ \\
$\ldots$ & 85.028 & $\mathrm{C}_{4} \mathrm{H}_{5} \mathrm{O}_{2}{ }^{+}$ & $\mathrm{C} 3$ to C6 fragment of $\mathrm{M}-2\left(\mathrm{H}_{2} \mathrm{O}\right)$ \\
$\ldots$ & 102.031 & $\mathrm{C}_{4} \mathrm{H}_{6} \mathrm{O}_{3}{ }^{+}$ & $\mathrm{M}-\mathrm{C}_{2} \mathrm{H}_{4} \mathrm{O}_{2}$ ring fragment $-\left(\mathrm{H}_{2} \mathrm{O}\right)$ \\
$\ldots$ & 109.028 & $\mathrm{C}_{6} \mathrm{H}_{5} \mathrm{O}_{2}{ }^{+}$ & $\mathrm{M}-4\left(\mathrm{H}_{2} \mathrm{O}\right)+\mathrm{H}$ \\
$\ldots$ & 127.039 & $\mathrm{C}_{6} \mathrm{H}_{7} \mathrm{O}_{3}{ }^{+}$ & $\mathrm{M}-3\left(\mathrm{H}_{2} \mathrm{O}\right)+\mathrm{H}$ \\
$\ldots$ & 145.050 & $\mathrm{C}_{6} \mathrm{H}_{9} \mathrm{O}_{4}{ }^{+}$ & $\mathrm{M}-2\left(\mathrm{H}_{2} \mathrm{O}\right)+\mathrm{H}$ \\
$\ldots$ & 163.060 & $\mathrm{C}_{6} \mathrm{H}_{11} \mathrm{O}_{5}{ }^{+}$ & $\mathrm{M}-\left(\mathrm{H}_{2} \mathrm{O}\right)+\mathrm{H}$ \\
$\ldots$ & 181.071 & $\mathrm{C}_{6} \mathrm{H}_{13} \mathrm{O}_{6}{ }^{+}$ & $\mathrm{M}+\mathrm{H}$ \\
\hline
\end{tabular}

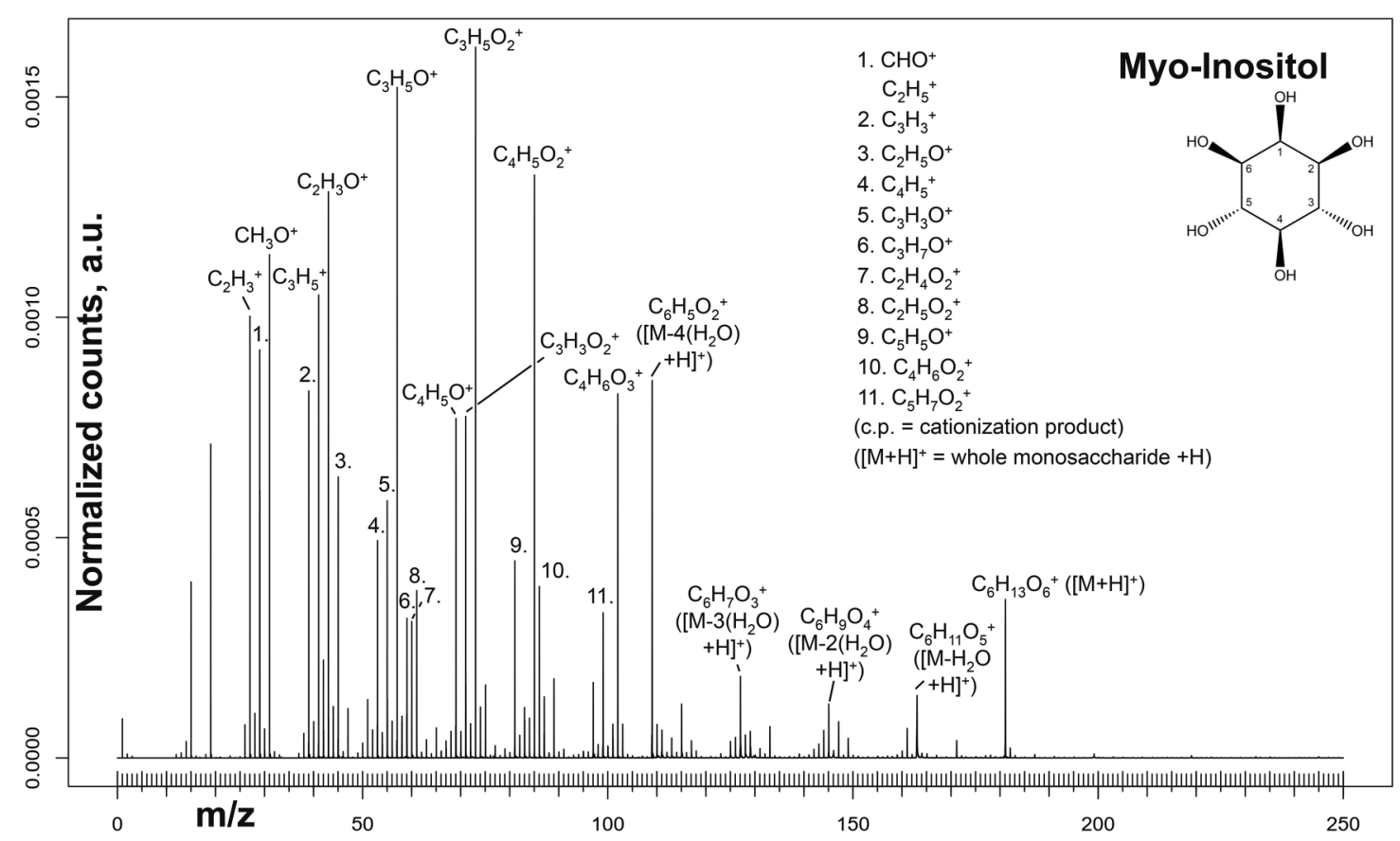

\begin{tabular}{rc}
\hline Accession \#: & $01602-01$ \\
\hline Host Material: & Silicon wafer \\
Technique: & SIMS \\
Secondary Source Polarity: & Positive \\
Mass Range: & $0-250 \mathrm{Da}$ \\
Species Used for Mass Calibration: & $\mathrm{CH}_{2}^{+}, \mathrm{CH}_{3}^{+}, \mathrm{C}_{2} \mathrm{H}_{4}^{+}, \mathrm{C}_{3} \mathrm{H}_{6}^{+}, \mathrm{C}_{2} \mathrm{H}_{4} \mathrm{O}^{+}$ \\
Primary lon Dose: & $9.71 \times 10^{11} \mathrm{~cm}^{-2}$ \\
Primary lon Pulse Width: & $17.2 \mathrm{~ns}$ \\
Pulsed Beam Current: & $0.00076 \mathrm{nA}$ \\
Biological Significance: & Myo-inositol derivatives serve as a Ca Ca secondary messenger; myo-inositol \\
& is also the basis for the phosphate storing molecule phytic acid in certain \\
& plants as well as the component of a wide range of phospholipids \\
\hline
\end{tabular}




\begin{tabular}{|c|c|c|c|}
\hline \multicolumn{4}{|c|}{ L-RHAMNOSE } \\
\hline Spectrum ID \# & Mass (Da) & Species & Peak Assignment \\
\hline 01603 & 71.049 & $\mathrm{C}_{4} \mathrm{H}_{7} \mathrm{O}^{+}$ & - \\
\hline$\ldots$ & 73.028 & $\mathrm{C}_{3} \mathrm{H}_{5} \mathrm{O}_{2}^{+}$ & - \\
\hline$\ldots$ & 75.044 & $\mathrm{C}_{3} \mathrm{H}_{7} \mathrm{O}_{2}^{+}$ & C4 to $\mathrm{C} 6$ fragment of $\mathrm{M}$ \\
\hline$\ldots$ & 85.028 & $\mathrm{C}_{4} \mathrm{H}_{5} \mathrm{O}_{2}^{+}$ & - \\
\hline$\ldots$ & 87.044 & $\mathrm{C}_{4} \mathrm{H}_{7} \mathrm{O}_{2}^{+}$ & C3 to $\mathrm{C} 6$ fragment of $\mathrm{M}-\left(\mathrm{H}_{2} \mathrm{O}\right)$ \\
\hline$\ldots$ & 89.023 & $\mathrm{C}_{3} \mathrm{H}_{5} \mathrm{O}_{3}^{+}$ & - \\
\hline$\ldots$ & 99.044 & $\mathrm{C}_{5} \mathrm{H}_{7} \mathrm{O}_{2}^{+}$ & $\mathrm{C} 2$ to $\mathrm{C} 6$ fragment of $\mathrm{M}-2\left(\mathrm{H}_{2} \mathrm{O}\right)$ \\
\hline$\ldots$ & 101.060 & $\mathrm{C}_{5} \mathrm{H}_{9} \mathrm{O}_{2}^{+}$ & $\mathrm{C} 2$ to $\mathrm{C} 6$ fragment of $\mathrm{M}-2\left(\mathrm{H}_{2} \mathrm{O}\right)+2 \mathrm{H}$ \\
\hline$\ldots$ & 103.039 & $\mathrm{C}_{4} \mathrm{H}_{7} \mathrm{O}_{3}^{+}$ & - \\
\hline$\ldots$ & 111.044 & $\mathrm{C}_{6} \mathrm{H}_{7} \mathrm{O}_{2}^{+}$ & $\mathrm{M}-3\left(\mathrm{H}_{2} \mathrm{O}\right)+\mathrm{H}$ \\
\hline$\ldots$ & 129.055 & $\mathrm{C}_{6} \mathrm{H}_{9} \mathrm{O}_{3}{ }^{+}$ & $\mathrm{M}-2\left(\mathrm{H}_{2} \mathrm{O}\right)+\mathrm{H}$ \\
\hline$\ldots$ & 147.065 & $\mathrm{C}_{6} \mathrm{H}_{11} \mathrm{O}_{4}^{+}$ & $\mathrm{M}-\left(\mathrm{H}_{2} \mathrm{O}\right)+\mathrm{H}$ \\
\hline$\ldots$ & 187.058 & {$\left[\mathrm{C}_{6} \mathrm{H}_{12} \mathrm{O}_{5}+\mathrm{Na}\right]^{+}$} & $\mathrm{M}+\mathrm{Na}$ \\
\hline
\end{tabular}

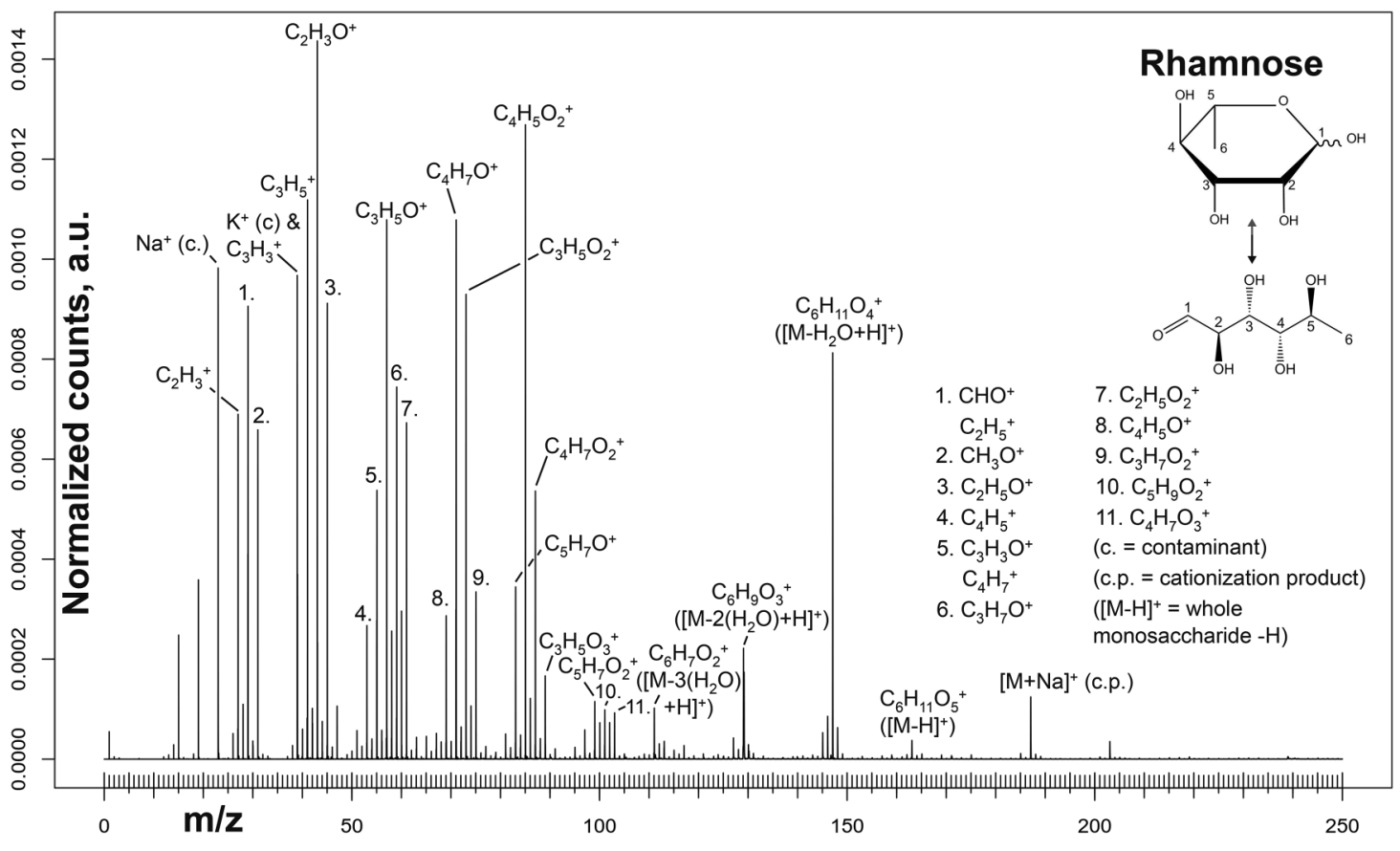

\begin{tabular}{rc}
\hline Accession \#: & $01603-01$ \\
\hline Host Material: & Silicon wafer \\
Technique: & SIMS \\
Secondary Source Polarity: & Positive \\
Mass Range: & $0-250 \mathrm{Da}$ \\
Species Used for Mass Calibration: & $\mathrm{CH}_{2}^{+}, \mathrm{CH}_{3}^{+}, \mathrm{C}_{2} \mathrm{H}_{4}^{+}, \mathrm{C}_{3} \mathrm{H}_{6}^{+}, \mathrm{C}_{2} \mathrm{H}_{4} \mathrm{O}^{+}$ \\
Primary lon Dose: & $9.71 \times 10^{11} \mathrm{~cm}^{-2}$ \\
Primary lon Pulse Width: & $17.2 \mathrm{~ns}$ \\
Pulsed Beam Current: & $0.00076 \mathrm{nA}$
\end{tabular}

Biological Significance:

L-Rhamnose is a component of the rhamnogalacturonan pectins as well as of certain bacterial outer cell membranes 


\begin{tabular}{lccc}
\hline & & D-RIBOSE \\
\hline Spectrum ID \# & Mass (Da) & Species & Peak Assignment \\
\hline 01604 & 73.028 & $\mathrm{C}_{3} \mathrm{H}_{5} \mathrm{O}_{2}{ }^{+}$ & C3 to C5 fragment of $\mathrm{M}-\left(\mathrm{H}_{2} \mathrm{O}\right)$ \\
$\ldots$ & 85.028 & $\mathrm{C}_{4} \mathrm{H}_{5} \mathrm{O}_{2}{ }^{+}$ & $\mathrm{C} 4$ to $\mathrm{C} 5$ fragment of $\mathrm{M}-2\left(\mathrm{H}_{2} \mathrm{O}\right)$ \\
$\ldots$ & 89.023 & $\mathrm{C}_{3} \mathrm{H}_{5} \mathrm{O}_{3}{ }^{+}$ & $\mathrm{C} 1$ to $\mathrm{C}$ fragment of $\mathrm{M}+\mathrm{H}$ \\
$\ldots$ & 97.028 & $\mathrm{C}_{5} \mathrm{H}_{5} \mathrm{O}_{2}{ }^{+}$ & $\mathrm{M}-3\left(\mathrm{H}_{2} \mathrm{O}\right)+\mathrm{H}$ \\
$\ldots$ & 115.039 & $\mathrm{C}_{5} \mathrm{H}_{7} \mathrm{O}_{3}{ }^{+}$ & $\mathrm{M}-2\left(\mathrm{H}_{2} \mathrm{O}\right)+\mathrm{H}$ \\
$\ldots$ & 133.050 & $\mathrm{C}_{5} \mathrm{H}_{9} \mathrm{O}_{4}{ }^{+}$ & $\mathrm{M}-\left(\mathrm{H}_{2} \mathrm{O}\right)+\mathrm{H}$ \\
\hline
\end{tabular}

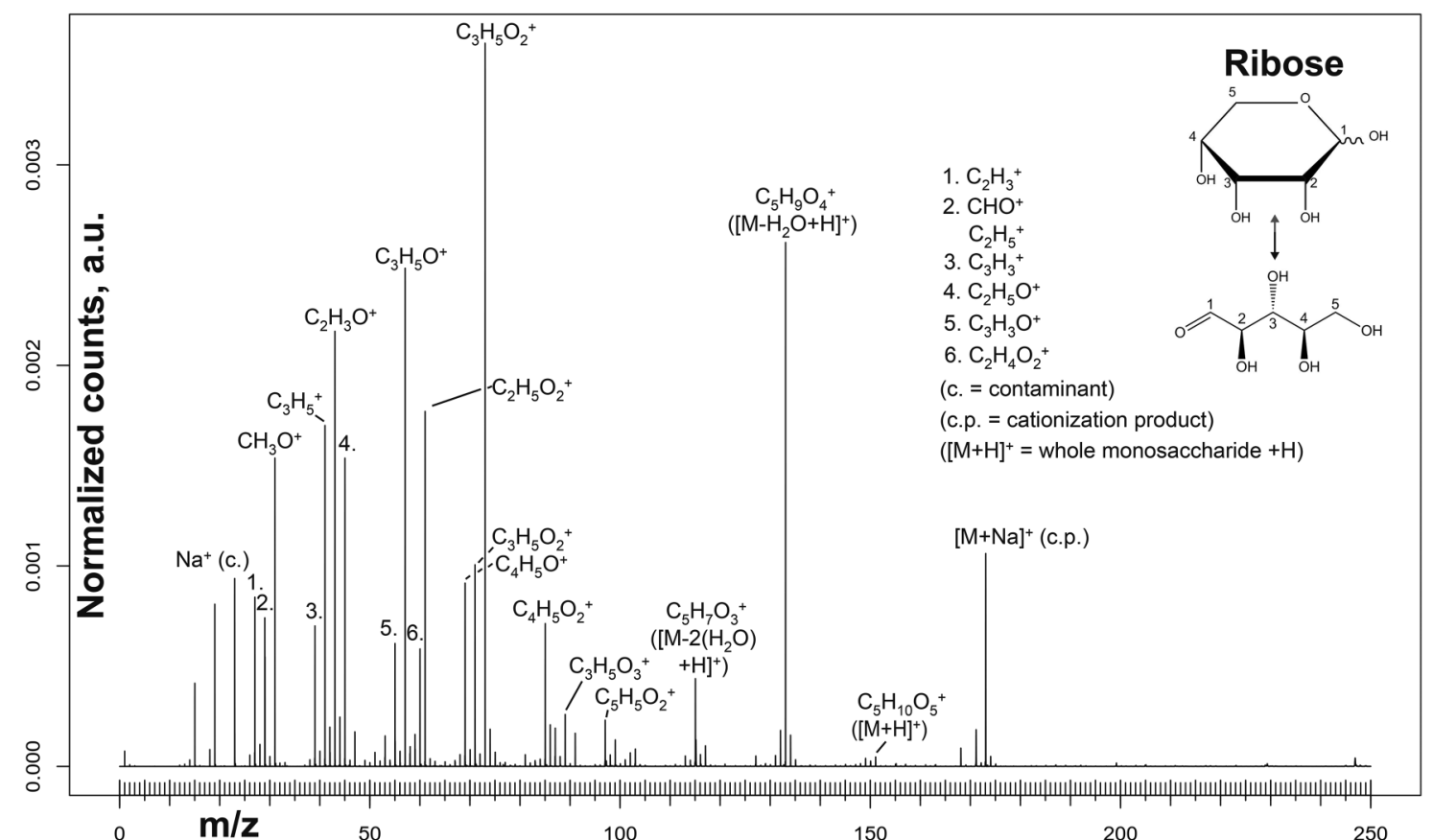

\section{Accession \#:}

Host Material:

Technique:

Secondary Source Polarity:

Mass Range:

Species Used for Mass

Calibration:

Primary Ion Dose:

Primary lon Pulse Width:

Pulsed Beam Current:

Biological Significance:

\section{4-01}

\section{Silicon wafer}

SIMS

Positive

0-250 Da

$$
\mathrm{CH}_{2}{ }^{+}, \mathrm{CH}_{3}{ }^{+}, \mathrm{C}_{2} \mathrm{H}_{4}{ }^{+}, \mathrm{C}_{3} \mathrm{H}_{6}{ }^{+}, \mathrm{C}_{2} \mathrm{H}_{4} \mathrm{O}^{+}
$$

$$
9.71 \times 10^{11} \mathrm{~cm}^{-2}
$$

$17.2 \mathrm{~ns}$

$0.00076 \mathrm{nA}$

D-Ribose (as $\alpha$-D-ribofuranose) forms the backbone of RNA and is, among others, part of the molecules ATP (adenosine triphosphate), ADP (adenosine diphosphate), and NAD (nicotinamide adenine dinucleotide) essential for the metabolism of all living cells 


\section{2-DEOXY-D-RIBOSE}

\begin{tabular}{lccc}
\hline Spectrum ID \# & Mass (Da) & Species & Peak Assignment \\
\hline 01605 & 57.033 & $\mathrm{C}_{3} \mathrm{H}_{5} \mathrm{O}^{+}$ & - \\
$\ldots$ & 69.033 & $\mathrm{C}_{4} \mathrm{H}_{5} \mathrm{O}^{+}$ & - \\
$\ldots$ & 73.065 & $\mathrm{C}_{4} \mathrm{H}_{9} \mathrm{O}^{+}$ & - \\
$\ldots$ & 81.033 & $\mathrm{C}_{5} \mathrm{H}_{5} \mathrm{O}^{+}$ & $\mathrm{M}-3\left(\mathrm{H}_{2} \mathrm{O}\right)+\mathrm{H}$ \\
$\ldots$ & 99.044 & $\mathrm{C}_{5} \mathrm{H}_{7} \mathrm{O}_{2}{ }^{+}$ & $\mathrm{M}-2\left(\mathrm{H}_{2} \mathrm{O}\right)+\mathrm{H}$ \\
$\ldots$ & 117.06 & $\mathrm{C}_{5} \mathrm{H}_{9} \mathrm{O}_{3}$ & $\mathrm{M}-\left(\mathrm{H}_{2} \mathrm{O}\right)+\mathrm{H}$ \\
\hline
\end{tabular}

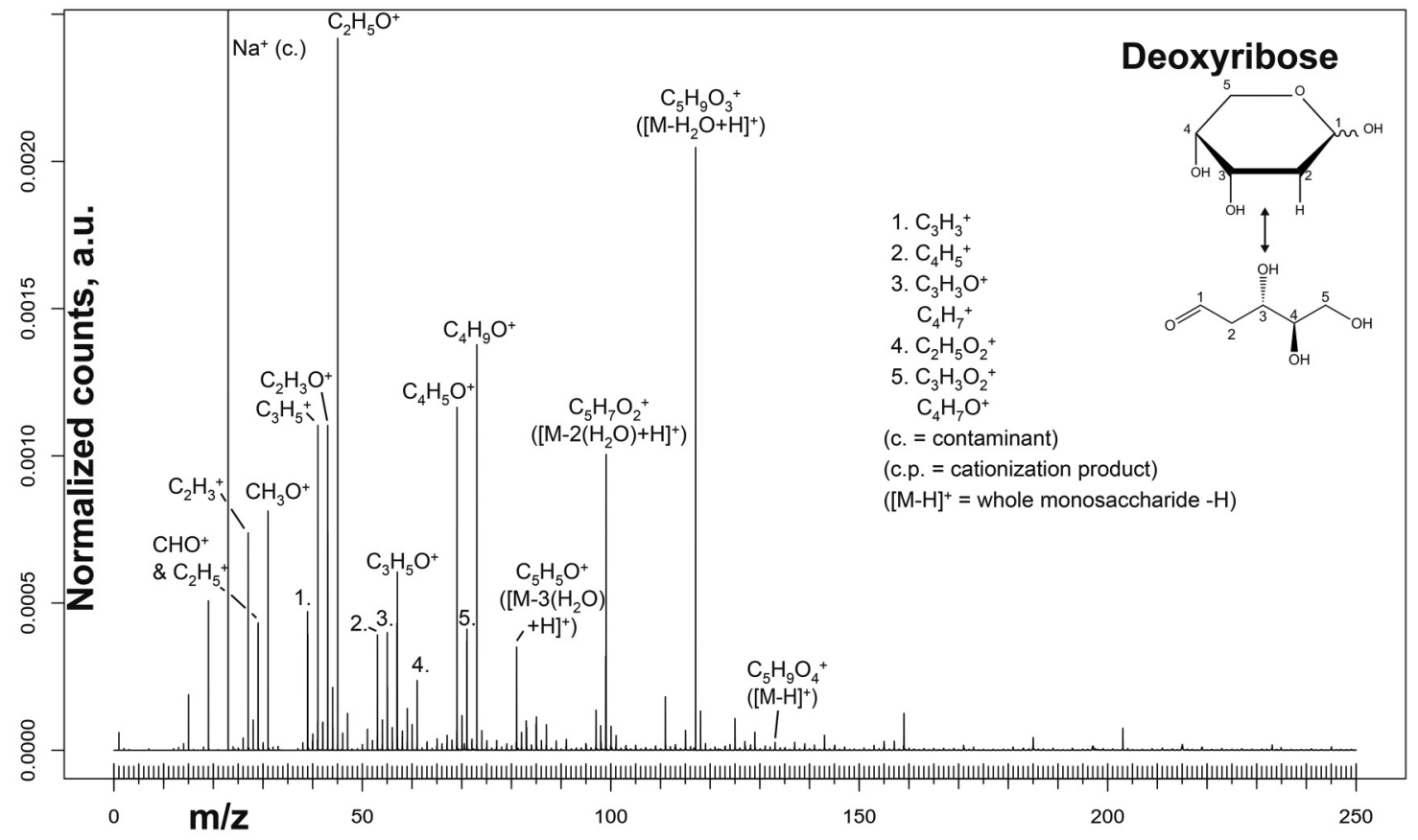

\begin{tabular}{rc}
\hline Accession \#: & $01605-01$ \\
\hline Host Material: & Technique: \\
Tecondary Source Polarity: & SIMS \\
Mass Range: & Positive \\
Species Used for Mass Calibration: & $0-250 \mathrm{Da}$ \\
Primary lon Dose: & $\mathrm{CH}_{2}^{+}, \mathrm{CH}_{3}^{+}, \mathrm{C}_{2} \mathrm{H}_{4}^{+}, \mathrm{C}_{3} \mathrm{H}_{6}^{+}, \mathrm{C}_{2} \mathrm{H}_{4} \mathrm{O}^{+}$ \\
Primary lon Pulse Width: & $9.71 \times 10^{11} \mathrm{~cm}^{-2}$ \\
Pulsed Beam Current: & $17.2 \mathrm{~ns}$ \\
Biological Significance: & $0.00076 \mathrm{nA}$ \\
\hline
\end{tabular}




\begin{tabular}{lccc}
\hline & & D-XYLOSE & \\
\hline Spectrum ID \# & Mass (Da) & Species & Peak Assignment \\
\hline 01606 & 73.028 & $\mathrm{C}_{3} \mathrm{H}_{5} \mathrm{O}_{2}{ }^{+}$ & $\mathrm{C} 3$ to C5 fragment of $\mathrm{M}-\left(\mathrm{H}_{2} \mathrm{O}\right)$ \\
$\cdots$ & 85.028 & $\mathrm{C}_{4} \mathrm{H}_{5} \mathrm{O}_{2}{ }^{+}$ & C4 to C5 fragment of $\mathrm{M}-2\left(\mathrm{H}_{2} \mathrm{O}\right)$ \\
$\cdots$ & 97.028 & $\mathrm{C}_{5} \mathrm{H}_{5} \mathrm{O}_{2}{ }^{+}$ & $\mathrm{M}-3\left(\mathrm{H}_{2} \mathrm{O}\right)+\mathrm{H}$ \\
$\cdots$ & 115.039 & $\mathrm{C}_{5} \mathrm{H}_{7} \mathrm{O}_{3}{ }^{+}$ & $\mathrm{M}-2\left(\mathrm{H}_{2} \mathrm{O}\right)+\mathrm{H}$ \\
$\cdots$ & 133.050 & $\mathrm{C}_{5} \mathrm{H}_{9} \mathrm{O}_{4}{ }^{+}$ & $\mathrm{M}-\left(\mathrm{H}_{2} \mathrm{O}\right)+\mathrm{H}$ \\
\hline
\end{tabular}

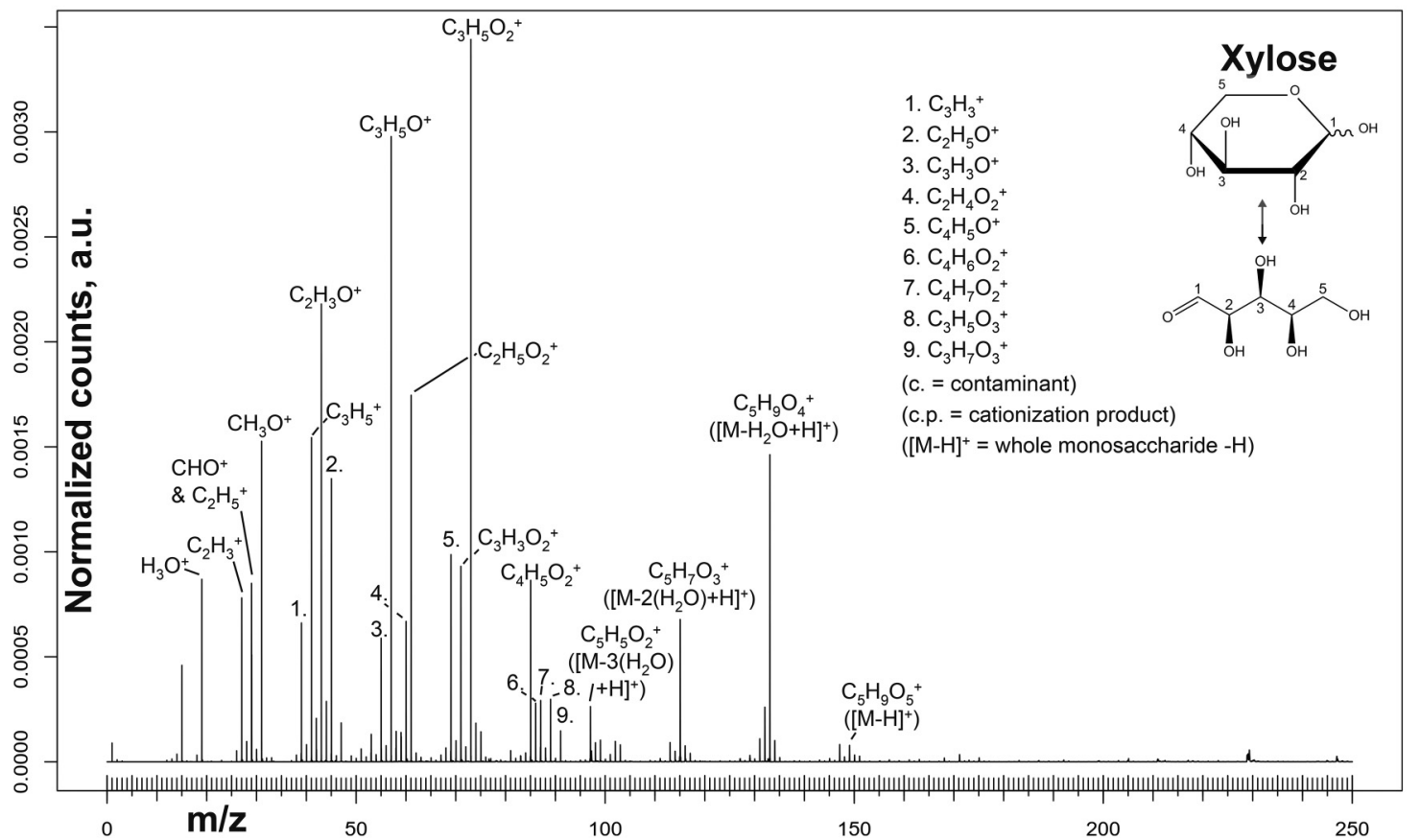

\begin{tabular}{rc}
\hline Accession \#: & $01606-01$ \\
\hline Host Material: & Silicon wafer \\
Technique: & SIMS \\
Mass Range: & Positive \\
Secondary Source Polarity: & $0-250 \mathrm{Da}$ \\
Species Used for Mass Calibration: & $\mathrm{CH}_{2}^{+}, \mathrm{CH}_{3}^{+}, \mathrm{C}_{2} \mathrm{H}_{4}^{+}, \mathrm{C}_{3} \mathrm{H}_{6}{ }^{+}, \mathrm{C}_{2} \mathrm{H}_{4} \mathrm{O}^{+}$ \\
Primary lon Dose: & $9.71 \times 10^{11} \mathrm{~cm}^{-2}$ \\
Primary lon Pulse Width: & $17.2 \mathrm{~ns}$ \\
Pulsed Beam Current: & $0.00076 \mathrm{nA}$ \\
Biological Significance: & D-Xylose is the main component of xylan, a highly abundant hemicellulose, \\
& and is, among others, also found as residue in certain types of pectin \\
\hline
\end{tabular}




\begin{tabular}{lccc}
\hline & \multicolumn{3}{c}{$\boldsymbol{N}$-ACETYL-D-GALACTOSAMINE } \\
\hline Spectrum ID \# & Mass (Da) & Species & Peak Assignment \\
\hline 01607 & 60.044 & $\mathrm{C}_{2} \mathrm{H}_{6} \mathrm{NO}^{+}$ & Amide group \\
$\ldots$ & 72.044 & $\mathrm{C}_{3} \mathrm{H}_{6} \mathrm{NO}^{+}$ & $\mathrm{C} 2+$ amide group \\
$\ldots$ & 84.044 & $\mathrm{C}_{4} \mathrm{H}_{6} \mathrm{NO}^{+}$ & - \\
$\ldots$ & 100.039 & $\mathrm{C}_{4} \mathrm{H}_{6} \mathrm{NO}_{2}{ }^{+}$ & - \\
$\ldots$ & 114.055 & $\mathrm{C}_{5} \mathrm{H}_{8} \mathrm{NO}_{2}{ }^{+}$ & - \\
$\ldots$ & 126.055 & $\mathrm{C}_{6} \mathrm{H}_{8} \mathrm{NO}_{2}{ }^{+}$ & $\mathrm{M}-3\left(\mathrm{H}_{2} \mathrm{O}\right)-\left(\mathrm{C}_{2} \mathrm{H}_{3} \mathrm{O}\right)$ at amide group $+2 \mathrm{H}$ \\
$\ldots$ & 138.055 & $\mathrm{C}_{7} \mathrm{H}_{8} \mathrm{NO}_{2}{ }^{+}$ & $\mathrm{M}-3\left(\mathrm{H}_{2} \mathrm{O}\right)-\mathrm{C}_{6}$ containing side group $+2 \mathrm{H}$ \\
$\ldots$ & 144.066 & $\mathrm{C}_{6} \mathrm{H}_{10} \mathrm{NO}_{3}{ }^{+}$ & $\mathrm{M}-2\left(\mathrm{H}_{2} \mathrm{O}\right)-\left(\mathrm{C}_{2} \mathrm{H}_{3} \mathrm{O}\right)$ at amide group $+2 \mathrm{H}$ \\
$\ldots$ & 162.076 & $\mathrm{C}_{6} \mathrm{H}_{12} \mathrm{NO}_{4}{ }^{+}$ & $\mathrm{M}-\left(\mathrm{H}_{2} \mathrm{O}\right)-\left(\mathrm{C}_{2} \mathrm{H}_{3} \mathrm{O}\right)$ at amide group $+2 \mathrm{H}$ \\
$\ldots$ & 186.076 & $\mathrm{C}_{8} \mathrm{H}_{12} \mathrm{NO}_{4}{ }^{+}$ & $\mathrm{M}-2\left(\mathrm{H}_{2} \mathrm{O}\right)+\mathrm{H}$ \\
$\ldots$ & 204.087 & $\mathrm{C}_{8} \mathrm{H}_{14} \mathrm{NO}_{5}{ }^{+}$ & $\mathrm{M}-\left(\mathrm{H}_{2} \mathrm{O}\right)+\mathrm{H}$ \\
\hline
\end{tabular}

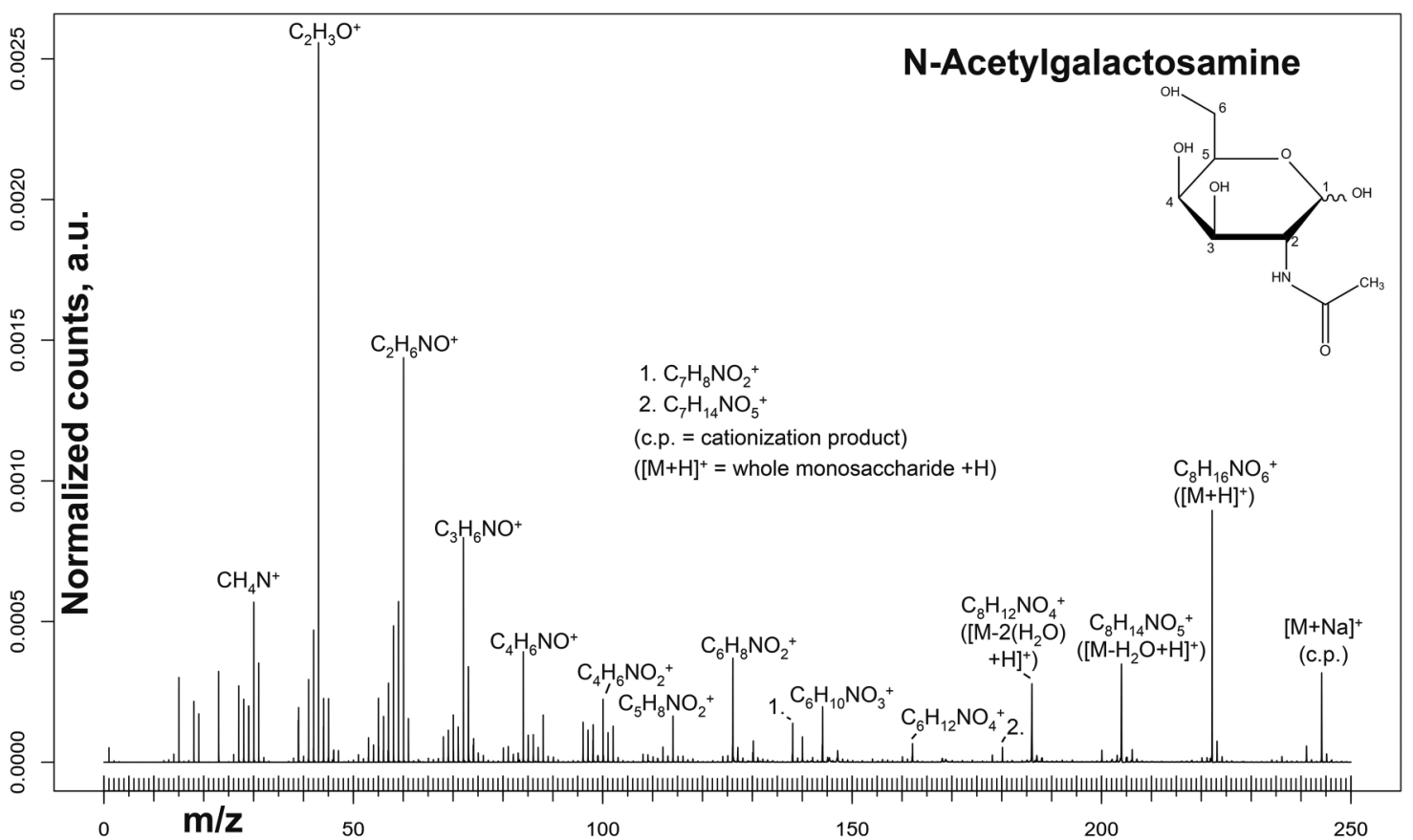

\begin{tabular}{rc}
\hline Accession \#: & $01607-01$ \\
\hline Host Material: \\
Technique: & Silicon wafer \\
Mass Range: & SIMS \\
Secondary Source Polarity: & Positive \\
Species Used for Mass Calibration: & $0-250 \mathrm{Da}$ \\
Primary lon Dose: & $\mathrm{CH}_{2}{ }^{+}, \mathrm{CH}_{3}^{+}, \mathrm{C}_{2} \mathrm{H}_{4} \mathrm{O}^{+}, \mathrm{C}_{8} \mathrm{H}_{16} \mathrm{NO}_{6}{ }^{+}$ \\
Primary lon Pulse Width: & $9.71 \times 10^{11} \mathrm{~cm}^{-2}$ \\
Pulsed Beam Current: & $17.2 \mathrm{~ns}$ \\
Biological Significance: & $0.00076 \mathrm{nA}$ \\
& $N$-acetylgalactosamine is found in a wide range of mucoproteins \\
& where it often serves as a linking point between the protein \\
& backbone and its covalently attached polysaccharide chains \\
\hline
\end{tabular}




\begin{tabular}{lccc}
\hline & \multicolumn{3}{c}{ N-ACETYL-D-GLUCOSAMINE } \\
\hline Spectrum ID \# & Mass (Da) & Species & Peak Assignment \\
\hline 01608 & 60.044 & $\mathrm{C}_{2} \mathrm{H}_{6} \mathrm{NO}^{+}$ & Amide group \\
$\ldots$ & 72.044 & $\mathrm{C}_{3} \mathrm{H}_{6} \mathrm{NO}^{+}$ & $\mathrm{C} 2+$ amide group \\
$\ldots$ & 84.044 & $\mathrm{C}_{4} \mathrm{H}_{6} \mathrm{NO}^{+}$ & - \\
$\ldots$ & 100.039 & $\mathrm{C}_{4} \mathrm{H}_{6} \mathrm{NO}_{2}{ }^{+}$ & - \\
$\ldots$ & 114.055 & $\mathrm{C}_{5} \mathrm{H}_{8} \mathrm{NO}_{2}{ }^{+}$ & - \\
$\ldots$ & 126.055 & $\mathrm{C}_{6} \mathrm{H}_{8} \mathrm{NO}_{2}{ }^{+}$ & $\mathrm{M}-3\left(\mathrm{H}_{2} \mathrm{O}\right)-\left(\mathrm{C}_{2} \mathrm{H}_{3} \mathrm{O}\right)$ at amide group $+2 \mathrm{H}$ \\
$\ldots$ & 138.055 & $\mathrm{C}_{7} \mathrm{H}_{8} \mathrm{NO}_{2}{ }^{+}$ & $\mathrm{M}-3\left(\mathrm{H}_{2} \mathrm{O}\right)-\mathrm{C}_{6}$ containing side group $+2 \mathrm{H}$ \\
$\ldots$ & 144.066 & $\mathrm{C}_{6} \mathrm{H}_{10} \mathrm{NO}_{3}{ }^{+}$ & $\mathrm{M}-2\left(\mathrm{H}_{2} \mathrm{O}\right)-\left(\mathrm{C}_{2} \mathrm{H}_{3} \mathrm{O}\right)$ at amide group $+2 \mathrm{H}$ \\
$\ldots$ & 162.076 & $\mathrm{C}_{6} \mathrm{H}_{12} \mathrm{NO}_{4}{ }^{+}$ & $\mathrm{M}-\left(\mathrm{H}_{2} \mathrm{O}\right)-\left(\mathrm{C}_{2} \mathrm{H}_{3} \mathrm{O}\right)$ at amide group $+2 \mathrm{H}$ \\
$\ldots$ & 186.076 & $\mathrm{C}_{8} \mathrm{H}_{12} \mathrm{NO}_{4}{ }^{+}$ & $\mathrm{M}-2\left(\mathrm{H}_{2} \mathrm{O}\right)+\mathrm{H}$ \\
$\ldots$ & 204.087 & $\mathrm{C}_{8} \mathrm{H}_{14} \mathrm{NO}_{5}{ }^{+}$ & $\mathrm{M}-\left(\mathrm{H}_{2} \mathrm{O}\right)+\mathrm{H}$ \\
\hline
\end{tabular}

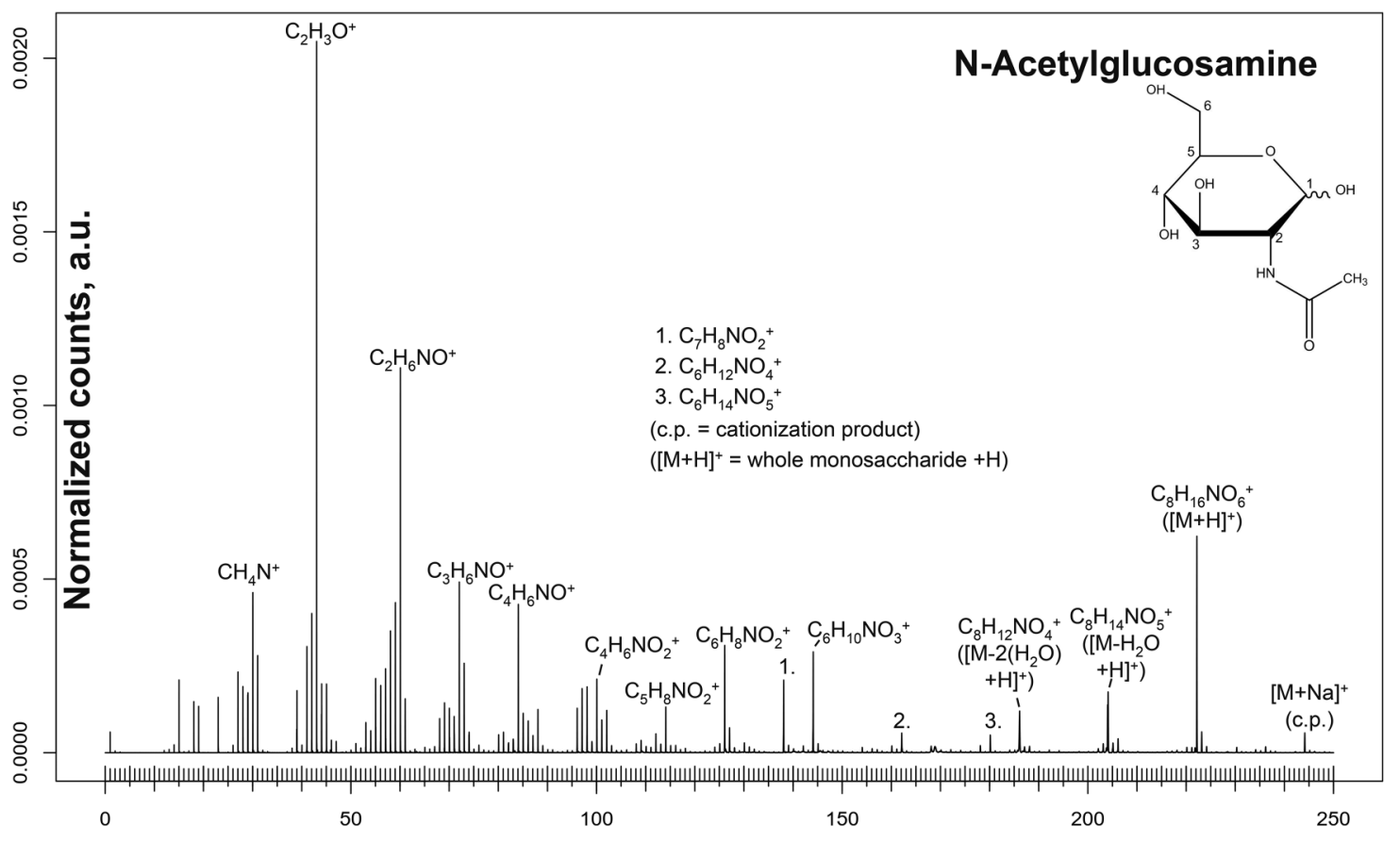

\begin{tabular}{rc}
\hline Accession \#: & $01608-01$ \\
\hline $\begin{array}{r}\text { Host Material: } \\
\text { Technique: }\end{array}$ & Silicon wafer \\
Mass Range: & SIMS \\
Secondary Source Polarity: & Positive \\
Species Used for Mass Calibration: & $0-250 \mathrm{Da}$ \\
Primary lon Dose: & $\mathrm{CH}_{2}{ }^{+}, \mathrm{CH}_{3}^{+}, \mathrm{C}_{2} \mathrm{H}_{4} \mathrm{O}^{+}, \mathrm{C}_{8} \mathrm{H}_{16} \mathrm{NO}_{6}{ }^{+}$ \\
Primary lon Pulse Width: & $9.71^{*} 10^{11} \mathrm{~cm}^{-2}$ \\
Pulsed Beam Current: & $17.2 \mathrm{~ns}$ \\
Biological Significance: & $N$-acetyl-D-glucosamine is the monomer unit of chitin, a polysaccharide that is, among \\
& others, the primary components of arthropodal exoskeletons and fungal cell walls. \\
& Independently, alternating covalently bound $N$-acetyl-D-glucosamine and \\
& $N$-acetylmuramic acid units form the amino sugar chains in the peptidoglycan, the \\
& bacterial cell wall. $N$-acetyl-D-glucosamine is also found, among others, in mucoproteins \\
\hline &
\end{tabular}




\begin{tabular}{lccc}
\hline & & N-ACETYLMURAMIC ACID & \\
\hline Spectrum ID \# & Mass (Da) & Species & Peak Assignment \\
\hline 01609 & 60.044 & $\mathrm{C}_{2} \mathrm{H}_{6} \mathrm{NO}^{+}$ & Amide group \\
$\ldots$ & 72.044 & $\mathrm{C}_{3} \mathrm{H}_{6} \mathrm{NO}^{+}$ & $\mathrm{C} 2+$ amide group \\
$\ldots$ & 84.044 & $\mathrm{C}_{4} \mathrm{H}_{6} \mathrm{NO}^{+}$ & - \\
$\ldots$ & 98.060 & $\mathrm{C}_{5} \mathrm{H}_{8} \mathrm{NO}^{+}$ & - \\
$\ldots$ & 100.039 & $\mathrm{C}_{4} \mathrm{H}_{6} \mathrm{NO}_{2}^{+}$ & - \\
$\ldots$ & 114.055 & $\mathrm{C}_{5} \mathrm{H}_{8} \mathrm{NO}_{2}^{+}$ & - \\
$\ldots$ & 126.055 & $\mathrm{C}_{6} \mathrm{H}_{8} \mathrm{NO}_{2}{ }^{+}$ & - \\
$\ldots$ & 138.055 & $\mathrm{C}_{7} \mathrm{H}_{8} \mathrm{NO}_{2}{ }^{+}$ & - \\
$\ldots$ & 144.066 & $\mathrm{C}_{6} \mathrm{H}_{10} \mathrm{NO}_{3}{ }^{+}$ & - \\
$\ldots$ & 186.076 & $\mathrm{C}_{8} \mathrm{H}_{12} \mathrm{NO}_{4}^{+}$ & - \\
$\ldots$ & 252.108 & $\mathrm{C}_{9} \mathrm{H}_{18} \mathrm{NO}_{7}^{+}$ & $\mathrm{C}$ \\
$\ldots$ & 276.108 & $\mathrm{C}_{11} \mathrm{H}_{18} \mathrm{NO}_{7}^{+}$ & $\mathrm{M}-\left(\mathrm{C}_{2} \mathrm{H}_{3} \mathrm{O}\right)$ at amide group $+2 \mathrm{H}$ \\
$\ldots$ & 294.118 & $\mathrm{C}_{11} \mathrm{H}_{20} \mathrm{NO}_{8}^{+}$ & $\mathrm{M}-\left(\mathrm{H}_{2} \mathrm{O}\right)+\mathrm{H}$ \\
\hline
\end{tabular}

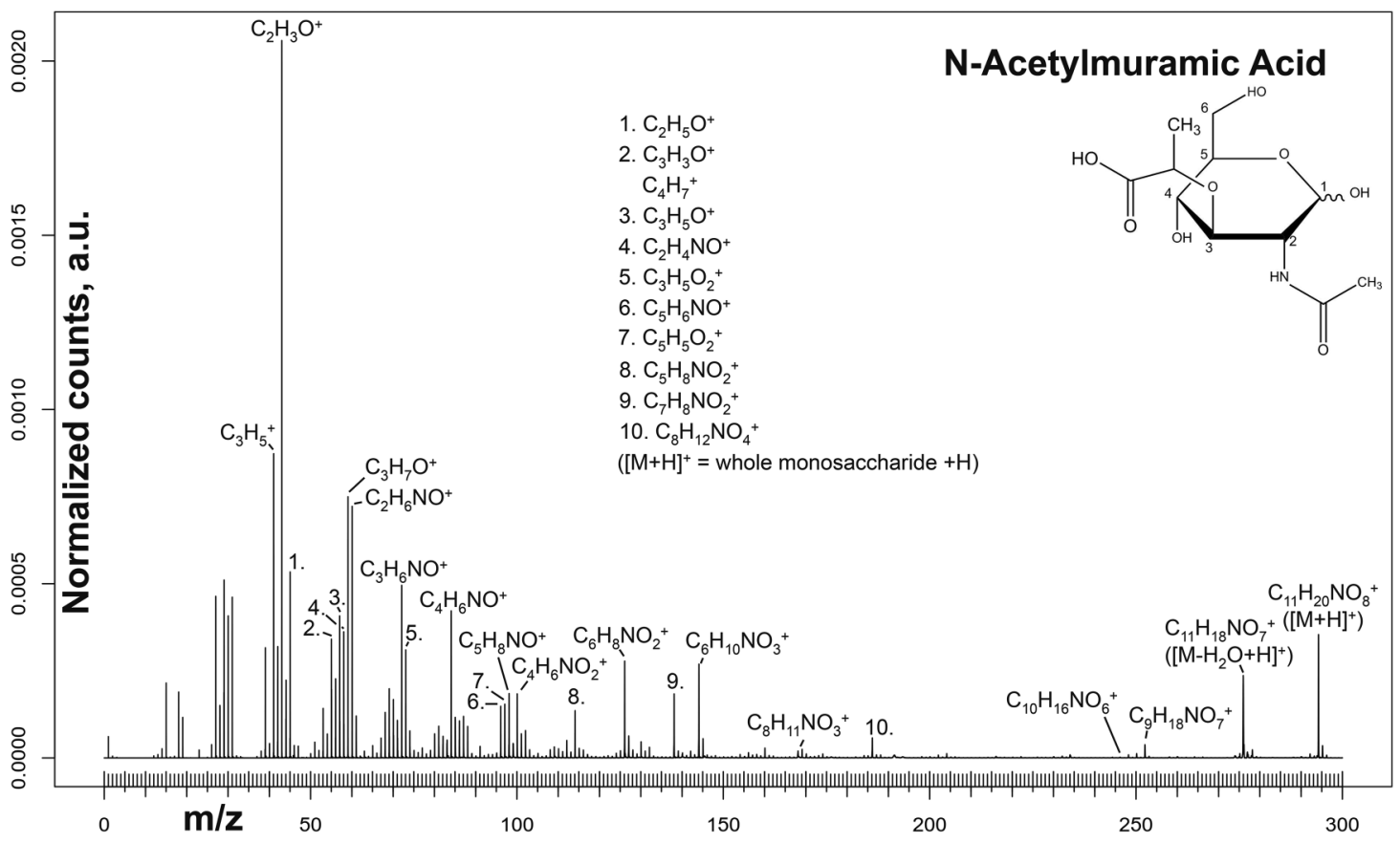

\begin{tabular}{|c|c|}
\hline Accession \#: & 01609-01 \\
\hline Host Material: & Silicon wafer \\
\hline Technique: & SIMS \\
\hline Secondary Source Polarity: & Positive \\
\hline Mass Range: & $0-300 \mathrm{Da}$ \\
\hline Species Used for Mass Calibration: & $\mathrm{CH}_{2}^{+}, \mathrm{CH}_{3}{ }^{+}, \mathrm{C}_{2} \mathrm{H}_{4} \mathrm{O}^{+}, \mathrm{C}_{11} \mathrm{H}_{20} \mathrm{NO}_{8}^{+}$ \\
\hline Primary Ion Dose: & $9.71 \times 10^{11} \mathrm{~cm}^{-2}$ \\
\hline Primary lon Pulse Width: & $17.2 \mathrm{~ns}$ \\
\hline Pulsed Beam Current: & $0.00076 \mathrm{nA}$ \\
\hline Biological Significance: & $\begin{array}{l}\text { Alternating covalently bound } N \text {-acetylmuramic acid and } N \text {-acetyl-D-glucosamine } \\
\text { units form the amino sugar chains of the peptidoglycan, the bacterial cell wall }\end{array}$ \\
\hline
\end{tabular}




\section{N-ACETYLNEURAMINIC ACID}

\begin{tabular}{lccc}
\hline Spectrum ID \# & Mass (Da) & Species & Peak Assignment \\
\hline 01610 & 60.044 & $\mathrm{C}_{2} \mathrm{H}_{6} \mathrm{NO}^{+}$ & Amide group \\
$\ldots$ & 72.044 & $\mathrm{C}_{3} \mathrm{H}_{6} \mathrm{NO}^{+}$ & $\mathrm{C} 2+$ amide group \\
$\ldots$ & 84.044 & $\mathrm{C}_{4} \mathrm{H}_{6} \mathrm{NO}^{+}$ & - \\
$\ldots$ & 127.075 & $\mathrm{C}_{7} \mathrm{H}_{11} \mathrm{O}_{2}^{+}$ & - \\
$\ldots$ & 274.092 & $\mathrm{C}_{11} \mathrm{H}_{16} \mathrm{NO}_{7}^{+}$ & $\mathrm{M}-2\left(\mathrm{H}_{2} \mathrm{O}\right)+\mathrm{H}$ \\
$\ldots$ & 292.103 & $\mathrm{C}_{11} \mathrm{H}_{18} \mathrm{NO}_{8}^{+}$ & $\mathrm{M}-\left(\mathrm{H}_{2} \mathrm{O}\right)+\mathrm{H}$ \\
$\ldots$ & 310.113 & $\mathrm{C}_{11} \mathrm{H}_{20} \mathrm{NO}_{9}^{+}$ & $\mathrm{M}+\mathrm{H}$ \\
\hline
\end{tabular}

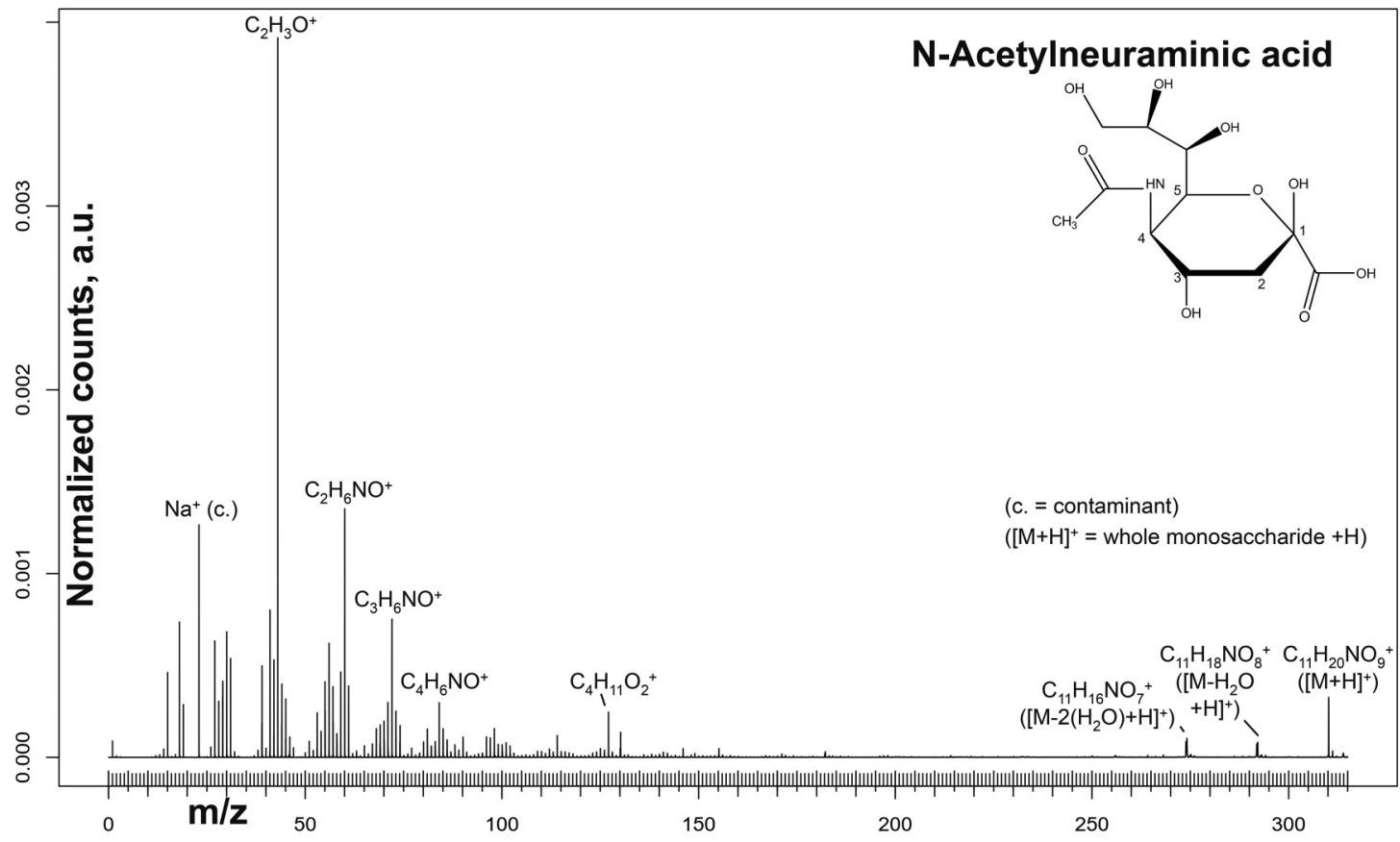

\begin{tabular}{rc}
\hline Accession \#: & $01610-01$ \\
\hline Host Material: & Silicon wafer \\
Technique: & SIMS \\
Mass Range: & Positive \\
Secondary Source Polarity: & $0-315 \mathrm{Da}$ \\
Primary lon Dose: & $\mathrm{CH}_{2}^{+}, \mathrm{CH}_{3}^{+}, \mathrm{C}_{2} \mathrm{H}_{4} \mathrm{O}^{+}, \mathrm{C}_{11} \mathrm{H}_{20} \mathrm{NO}_{9}{ }^{+}$ \\
Primary lon Pulse Width: & $9.71 \times 10^{11} \mathrm{~cm}^{-2}$ \\
Pulsed Beam Current: & $17.2 \mathrm{~ns}$ \\
Biological Significance: & $0.00076 \mathrm{nA}$ \\
& $N$-acetylneuraminic acid is found in a wide range of glycoproteins and is \\
& crucial in the modulation of neurosynaptic transmission in humans \\
\hline
\end{tabular}



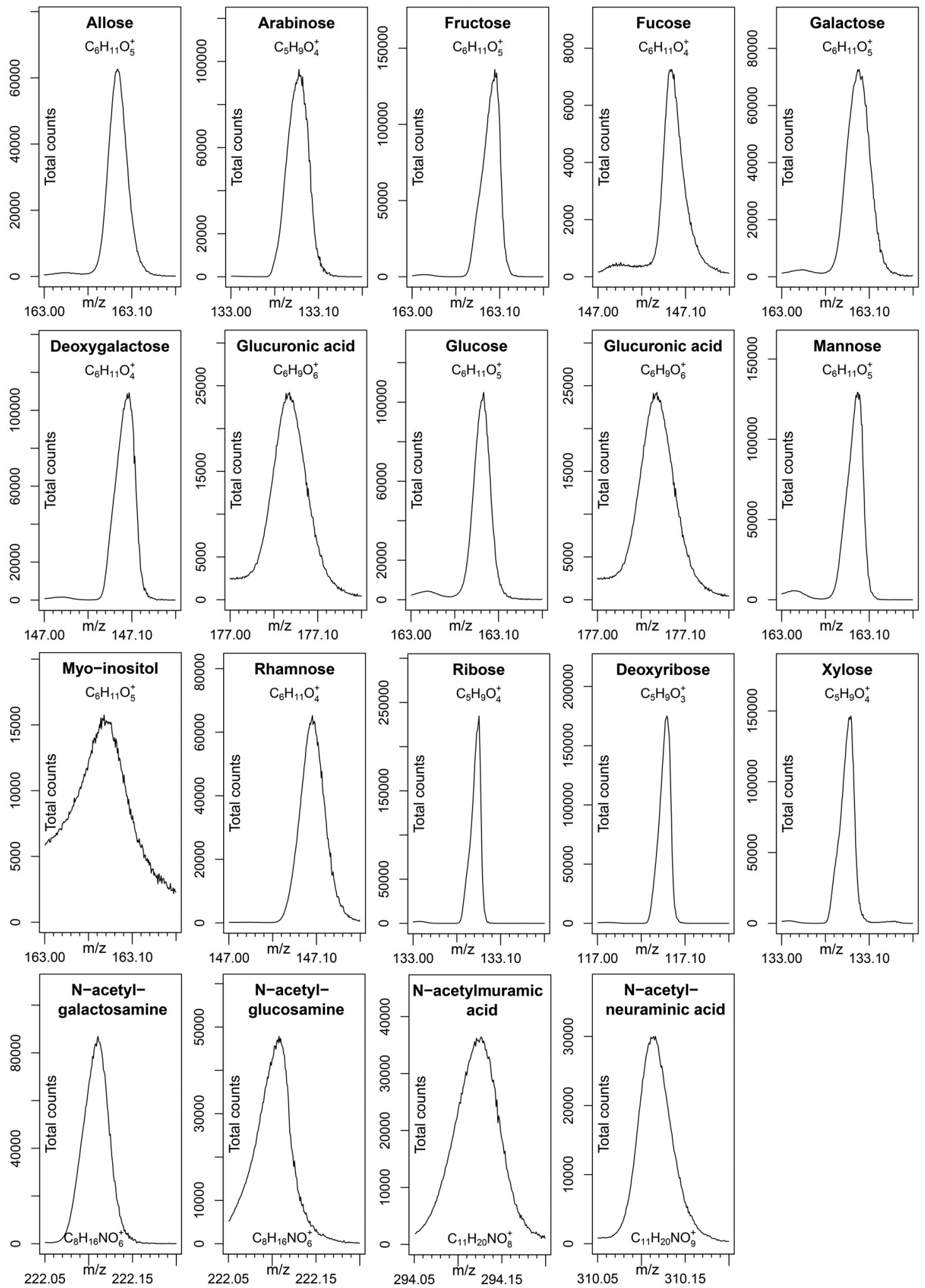

Accession \#:

01592-01, 01593-01, 01594-01, 01595-01, 01596-01, 01597-01, 01598-01, 01599-01, 01600-01, 01601-01, 01602-01, 01603-01, 01604-01, 01605-01, 01606-01, 01607-01, 01608-01, 01609-01, 01610-01 